\title{
Mapping the Cavity Optomechanical Interaction with Subwavelength-Sized Ultrasensitive Nanomechanical Force Sensors
}

\author{
Francesco Fogliano, ${ }^{1, *}$ Benjamin Besga, ${ }^{1, *}$ Antoine Reigue, ${ }^{1, *}$ Philip Heringlake, ${ }^{1}$ Laure Mercier de Lépinay® ${ }^{1}$ \\ Cyril Vaneph $\odot,{ }^{2}$ Jakob Reichel, ${ }^{2}$ Benjamin Pigeau, ${ }^{1}$ and Olivier Arcizet ${ }^{1, \dagger}$ \\ ${ }^{1}$ Institut Néel, Université Grenoble Alpes-CNRS:UPR2940, 38042 Grenoble, France \\ ${ }^{2}$ Laboratoire Kastler Brossel, ENS-Université PSL, CNRS, Sorbonne Université, \\ Collège de France, 75005 Paris, France
}

(Received 17 September 2020; revised 13 January 2021; accepted 12 February 2021; published 8 April 2021)

\begin{abstract}
In canonical optomechanical systems, mechanical vibrations are dynamically encoded on an optical probe field, which reciprocally exerts a backaction force. Because of the weak single-photon coupling strength achieved with macroscopic oscillators, most of the existing experiments were conducted with large photon numbers to achieve sizable effects, thereby hiding the original optomechanical nonlinearity. To increase the optomechanical interaction, we make use of subwavelength-sized ultrasensitive suspended nanowires inserted in the mode volume of a fiber-based microcavity. By scanning the nanowire within the cavity mode volume and measuring its impact on the cavity mode, we obtain a map of the 2D optomechanical interaction. Then, by using the toolbox of nanowire-based force-sensing protocols, we explore the backaction of the optomechanical interaction and map the optical force field experienced by the nanowire. These measurements also allow us to demonstrate the possibility to detect variations of the mean intracavity photon number smaller than unity. This implementation should also allow us to enter the promising regime of cavity optomechanics, where a single intracavity photon can displace the oscillator by more than its zero-point fluctuations, which will open novel perspectives in the field.
\end{abstract}

DOI: 10.1103/PhysRevX.11.021009

Subject Areas: Nanophysics, Optics, Quantum Physics

\section{INTRODUCTION}

The field of optomechanics has gone through many impressive developments over the last decades [1]. The coupling between a probe light field and a mechanical degree of freedom (namely, an oscillator), possibly assisted by a high-finesse cavity, was early proposed as an ideal platform to explore the quantum limits of ultrasensitive measurements, where the quantum fluctuations of light are the dominant source of measurement noise [2-5]. The measurement backaction was also employed to manipulate the oscillator state through optical forces and dynamical backaction, leading to optomechanical correlations between both components of the system. In this framework, ground-state cooling, mechanical detection of radiationpressure quantum noise, advanced correlation between light and mechanical states or optomechanical squeezing were reported [6-19].

\footnotetext{
*These authors contributed equally to this work. †olivier.arcizet@neel.cnrs.fr
}

Published by the American Physical Society under the terms of the Creative Commons Attribution 4.0 International license. Further distribution of this work must maintain attribution to the author(s) and the published article's title, journal citation, and DOI.
All of these impressive results were obtained in the linear regime of cavity optomechanics, making use of large photon numbers, where the interaction Hamiltonian is linearized around an operating set point. However, the optomechanical interaction possesses an intrinsic Kerr-like nonlinearity [20] operating down to the single-photon level, which has, for the moment, remained far from experimental reach due to the weak single-photon coupling strength achieved with macroscopic oscillators.

This regime is achieved when a single photon in the cavity pushes the static rest position of the mechanical resonator by a quantity $\delta x^{(1)}$, which is larger than its zeropoint fluctuations $\delta x_{\mathrm{zpf}}$. A very strong optomechanical interaction is indeed needed to fulfil this condition since it requires $g_{0}>\Omega_{\mathrm{m}}$, where $g_{0}$ is the single-photon optomechanical coupling and $\Omega_{\mathrm{m}}$ the resonant angular frequency of the mechanical oscillator. The above criteria is a static (vs dynamic) metric of the interaction strength, and while the terminology is not yet fully consistent across the literature, it represents a prerequisite to enter into the single-photon regime of cavity optomechanics, a major experimental challenge pursued by the community.

Depending on the relative scaling of the cavity linewidth, oscillator frequency, and single-photon coupling strength $\left(\kappa, \Omega_{\mathrm{m}}, g_{0}\right.$ ), different novel dynamical phenomena can be anticipated [21-32], leading, for example, to the possibility of 
observing a single-photon blockade [24] if the photon lifetime in the cavity is long enough $\left(g_{0}>\kappa\right)$ or cooling beyond the Lamb-Dicke regime. At present, only atom-based optomechanical experiments $[33,34]$ could approach such a regime, while recent developments with highly deformable photonic crystals [35,36] or trampoline [37] resonators represent interesting platforms in that perspective.

In our approach, we employ subwavelength-sized nanowires, which produce significant photon scattering out of the cavity mode, so we operate in the single-photon adiabatic regime $\left(\Omega_{\mathrm{m}} \ll g_{0} \ll \kappa\right)$, where the cavity field instantaneously follows mechanically induced perturbations. This regime opens perspectives for exploring the optomechanical interaction at the single mean intracavity photon level, which should be within experimental reach at cryogenic temperatures. It represents an interesting resource for quantum optics since the optomechanical interaction to the nanowire is sufficient to generate nonclassical states of light down to very small mean photon numbers and in a broad frequency range, not restricted to the vicinity of the mechanical resonance $[5,20]$. Furthermore, significant deviations from the commonly employed semiclassical description can be expected since the optical field fluctuations can have an optomechanical impact comparable to the mean field at low photon number, which should also open the road towards the investigation of vacuum Casimir forces in confined optical resonators.

In our experiment, we make use of an ultrasensitive force sensor, a suspended silicon carbide nanowire with subwavelength-sized diameter whose vibrating extremity is inserted in the optical mode volume of a high-finesse fiber

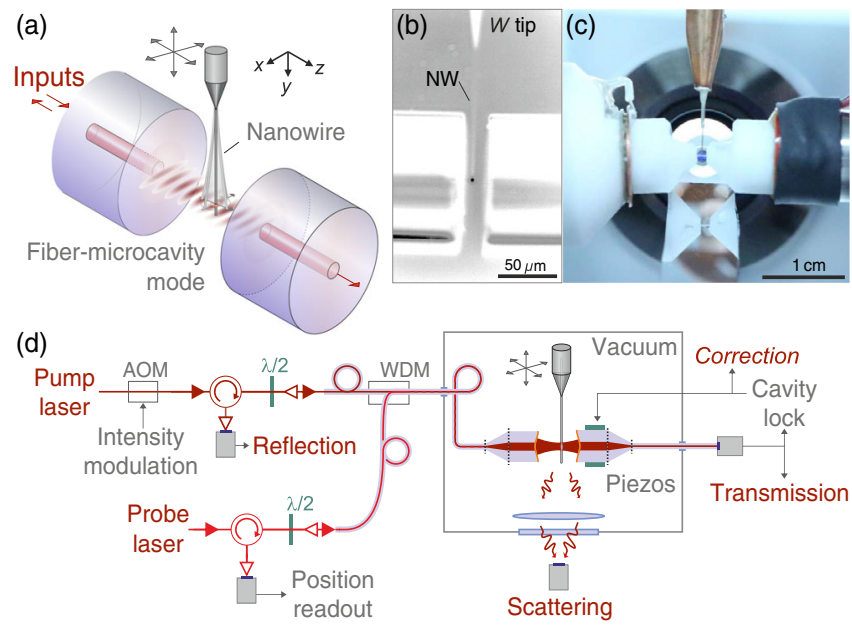

FIG. 1. Experimental setup. (a) Vibrating extremity of a silicon carbide nanowire piezo positioned in the optical mode volume of a high-finesse fiber microcavity to produce a large parametric coupling between the cavity optical mode and the oscillator position. (b,c) Lateral objective serving to align the experiment and collect part of the side-scattered light [dark spot in (b)]. (d) Sketch of the experiment. AOM: acousto-optic modulator; WDM: wavelength dividing module. microcavity [see Fig. 1(a)]. This "nanowire-in-the-middle" configuration decouples mechanics from optics $[8,9,11,17,38-46]$ and makes use of ultrasensitive nanowires as mechanical resonators [47-52] coupled to very-short-fiber Fabry-Pérot microcavities [53-55]. This experimental setup is versatile and allows us to study various aspects of the optomechanical interaction. We first explore the optical properties of the cavity field with a subwavelength-sized probe, using scanning probe imaging techniques. We measure the dependence on the nanowire position of the resonant frequency and linewidth of the cavity mode. This measurement allows us to spatially map the parametric optomechanical coupling strength, which acquires a vectorial character due to the ability of the nanowire to vibrate along both transverse directions in the cavity mode. This exploration allows us to identify locations within the cavity mode, close to the nodes, where the optomechanical coupling is maximized and where the light scattered out of the cavity mode by the nanowire, due to the enhanced light-nanowire interaction mediated by internal optical Mie resonances, has a reduced impact on the cavity linewidth. Then, we investigate the other facet of the optomechanical interaction and realize, for the first time, a full mapping of the optomechanical force experienced by the nanowire inside the cavity mode. Those force measurements are realized using pump-probe techniques [48], operating down to intensity modulation levels corresponding to less than one mean intracavity photon change. They provide a novel analytic tool of the intracavity field, complementary to optical measurements $[55,56]$.

Contrary to the case of canonical optomechanical model systems, those force measurements are essential here to fully understand the optomechanical interaction: It is not possible, in general, to obtain a complete knowledge of the electromagnetic field around the nanowire-and of the optical force it experiences-from a limited set of external optical measurements (transmission, reflection scatter channels and parametric coupling strength).

We then discuss the perspectives of this approach, or which allowing us to largely enter the emerging field of the single-photon and adiabatic regimes of cavity optomechanics and introduce some of the phenomena that would become accessible when working with cryogenically cooled nanowires.

\section{FORMALIZATION}

The optomechanical interaction between an optical cavity mode and a single mechanical vibration mode (pulsations $\omega_{0}$ and $\Omega_{\mathrm{m}}$; ladder operators $\hat{a}, \hat{a}^{\dagger}$ and $\hat{b}, \hat{b}^{\dagger}$, respectively) is canonically described by the coupling Hamiltonian $H_{\text {int }}=\hbar g_{0} \hat{a}^{\dagger} \hat{a}\left(\hat{b}+\hat{b}^{\dagger}\right)$, which formalizes the parametric dependence of the optical cavity resonance pulsation $\omega_{0}(\hat{x})$ on the oscillator position $\hat{x}=\delta x_{\mathrm{zpf}}\left(\hat{b}+\hat{b}^{\dagger}\right)$, where $\delta x_{\mathrm{zpf}}=$ $\sqrt{\hbar / 2 M_{\text {eff }} \Omega_{\mathrm{m}}}$ is the spatial spreading of the oscillator zeropoint fluctuations (effective mass $M_{\text {eff }}$ ). The single-photon 
parametric coupling strength $g_{0}=\partial_{x} \omega_{0} \delta x_{\mathrm{zpf}}$ is maximum for oscillators featuring large zero-point fluctuations-motivating the shift towards ultralight nanowires - interacting with small-mode-volume cavity modes. For a purely parametric coupling, a single-photonic excitation $\left(\left\langle\hat{a}^{\dagger} \hat{a}\right\rangle=1\right)$ generates an optical force of $F^{(1)}=-\hbar g_{0} / \delta x_{\mathrm{zpf}}$, which causes a static displacement of $\delta x^{(1)}=F^{(1)} / M \Omega_{\mathrm{m}}^{2}=$ $-2 g_{0} / \Omega_{\mathrm{m}} \delta x_{\mathrm{zpf}}$. As such, a single intracavity photon can be expected to have an appreciable impact on the oscillator state when $\delta x^{(1)}>\delta x_{\mathrm{zpf}}$, which requires $g_{0}>\Omega_{\mathrm{m}} / 2$. In the presence of position-dependent optical losses (dissipative coupling), the magnitude of the force may differ from the above expression, which underlines the importance of spatially mapping the optical force experienced by the oscillator.

Furthermore, this static deformation will, in turn, parametrically shift the cavity resonance by a quantity $\delta \omega^{(1)}=$ $g_{0} \delta x^{(1)} / \delta x_{\mathrm{zpf}}$. If it exceeds the cavity linewidth, $\delta \omega^{(1)}>\kappa$, the system can thus present a static optomechanical nonlinearity (bistability here) at the single intracavity photon level. Its observability thus requires large single-photon parametric cooperativities, $\mathcal{C}^{(1)} \equiv 2 g_{0}^{2} / \Omega_{\mathrm{m}} \kappa>1$.

Up to now, the achieved single-photon coupling strength $g_{0}$ remained too faint to have any impact of a single photon on the oscillator state, and all experiments were thus realized with large photon numbers to enhance the optomechanical interaction. In that situation, the system dynamics can be described by the linearized Hamiltonian: $\hbar g_{0} \bar{\alpha}\left(\delta \hat{a}+\delta \hat{a}^{\dagger}\right) \times$ $\left(\hat{b}+\hat{b}^{\dagger}\right)$, where the effective coupling strength $g_{0} \bar{\alpha}$ is formally enhanced by the mean intracavity field $\bar{\alpha}$. However, by doing so, the fundamental optomechanical nonlinearity (the optomechanically induced optical dephasing is proportional to the intracavity intensity like in the Kerr effect) gets diluted, and the regime of single-photon optomechanics remains, for the moment, out of experimental reach. Our approach is based on subwavelength-sized nanowires hosting internal optical Mie resonances [57,58] (which enhances the light-nanowire interaction), inserted in smallmode-volume microcavities, allowing us to achieve extremely large optomechanical interaction, at the condition to precisely microposition the nanowire extremity within the intracavity field. This approach thus requires a careful analysis of the spatial structure of the optomechanical interaction, which is a central result of this article.

\section{THE EXPERIMENT}

The fiber microcavity is made of two single-mode fibers with concavely laser-machined extremities covered with high-reflectivity dielectric coatings (approximately $28 \mu \mathrm{m}$ radii of curvature) [59]. The optical finesse can be adjusted from 400 to 45000 by tuning the laser wavelength from $760 \mathrm{~nm}$ to $820 \mathrm{~nm}$. The cavity geometry (fiber angles, lateral position, and coarse axial length) is adjusted using motorized supports, while the cavity length can be finely tuned using a set of piezo elements for fast and slow actuation (see the Appendix A). To minimize the optical mode volume, we operate with cavity lengths down to $10 \mu \mathrm{m}$ - which still allows us to safely insert the nanowire between the fibers [see Figs. 1(a) and 1(b)] - producing an optical cavity waist of $\omega_{0} \approx 1.8 \mu \mathrm{m}$. Our nanomechanical probes are silicon carbide nanowires [48-50,52], mounted on sharp metallic tips, whose vibrating extremity can be finely positioned within the cavity mode using an $X Y Z$ piezo stage. The employed nanowires oscillate in the $10-100 \mathrm{kHz}$ range, with effective masses around $1 \mathrm{pg}$ and subwavelength-sized diameters $(100-200 \mathrm{~nm})$. The experiment is conducted in static vacuum (below $10^{-2} \mathrm{mbar}$ ), where quality factors of around 5000 are achieved, in a suspended vacuum chamber to avoid vibrations and thermally insulated to improve its long-term stability. The microcavity is pumped by a low-noise tunable infrared Ti:saphire laser. We record the transmitted and reflected signals, but also the side-scattered photons, which are collected through a laterally positioned microscope objective [see Figs. 1(c) and 1(d)]. A digital feedback loop acting on the cavity piezos allows us to lock the cavity length on resonance.

\section{OPTOMECHANICAL COUPLING STRENGTH}

We first determine the parametric optomechanical coupling strength by measuring the dependence of the cavity resonance $\omega_{0}(\mathbf{r})$ on the nanowire position $\mathbf{r}$. To do so, we scan the cavity length with the slow piezo stack around a mean value of $L=12 \mu \mathrm{m}$ and insert the nanowire in the optical mode volume. Figure 2(a) represents the cavity transmission recorded when pumped at $767 \mathrm{~nm}$ in the vicinity of a $\mathrm{TEM}_{00}$ mode while scanning a 130 -nm-thick nanowire across the optical mode volume (along $\mathbf{e}_{\mathbf{x}}$ ). The nanowire causes a cavity frequency shift, a modification of its linewidth, and a change of the resonant transmission level. The nanowire diameter being much smaller than the optical wavelength, one recognizes the lateral Gaussian shape of the TEM ${ }_{00}$ cavity mode, with a fitted optical waist of $1.8 \mu \mathrm{m}$. When fully inserted (below the optical axis), the nanowire parametrically shifts the resonant cavity length by $\Delta L=-12 \mathrm{~nm}$, corresponding to an equivalent frequency shift of $\Delta \omega_{0}=-\omega_{0} \Delta L / L=2 \pi \times 400 \mathrm{GHz}$. It corresponds to a $10^{-3}$ relative cavity shift, in agreement with the relative increase of the optical mode volume (see Appendix C). Similar measurements can be realized while scanning the nanowire along the optical axis $\left(\mathbf{e}_{\mathbf{z}}\right)$ [see Fig. 2(c)], revealing the standing-wave structure of the cavity mode, with $\lambda / 2$ periodicity. When the nanowire is positioned on a node of the optical mode, the cavity field remains almost unperturbed.

Those measurements allow us to spatially map the parametric coupling strength. Since the nanowire can move identically along both transverse $(x z)$ directions, it is necessary to adopt a vectorial coupling strength $\left.\mathbf{G} \equiv \boldsymbol{\nabla} \omega_{0}\right|_{\mathbf{r}_{0}}$, which has to be evaluated at the nanowire rest position $\mathbf{r}_{\mathbf{0}}$. 
(a)

(b)
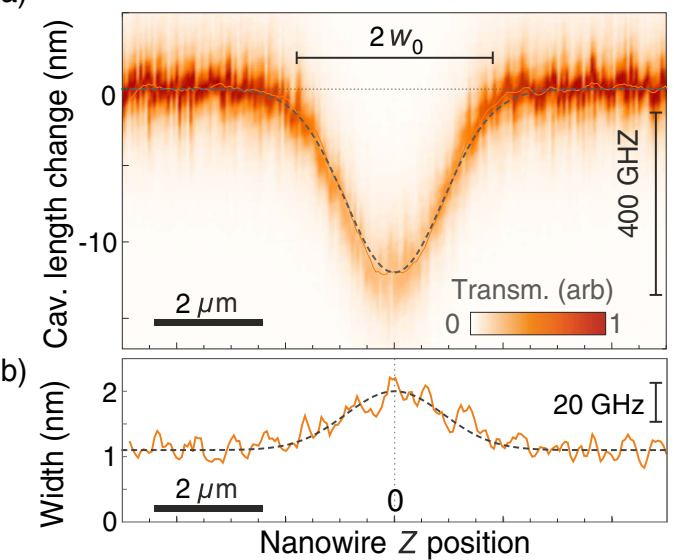

(c)

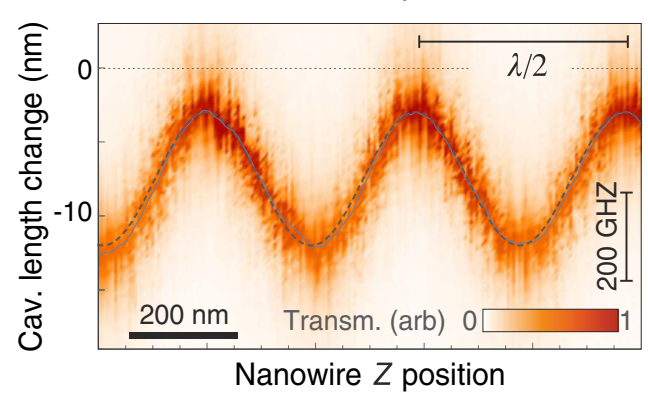

(d)
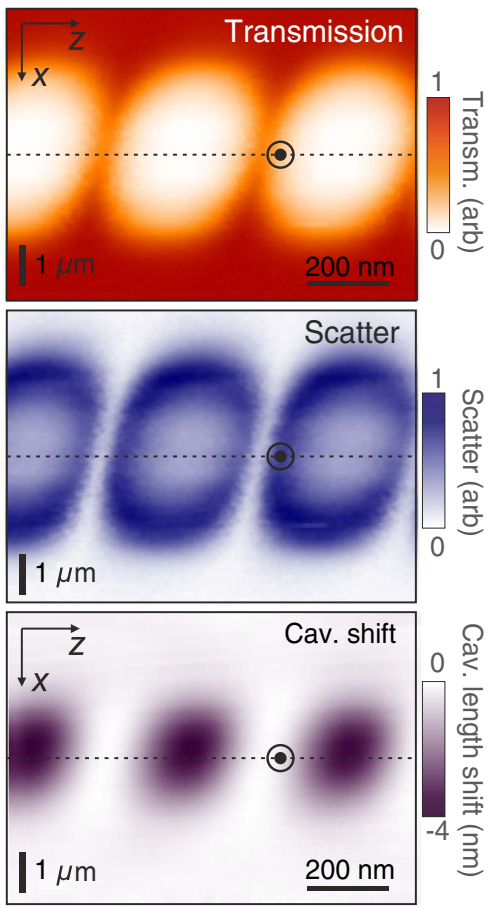

(e)

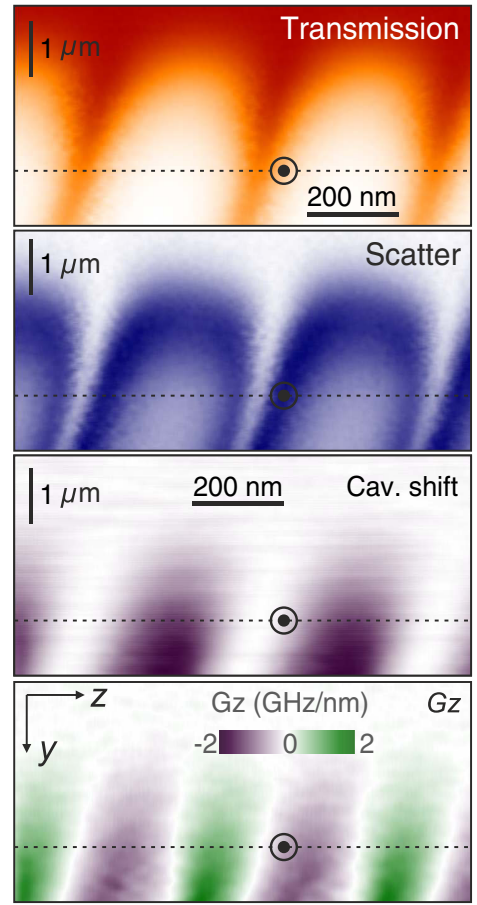

FIG. 2. Nanowire-based scanning probe exploration of the intracavity field. (a) Transmission stack of the cavity measured while scanning its total length with the slow piezo, in the vicinity of a TEM T0 $_{0}$ mode and moving the nanowire across the optical mode ( $x$ axis). The dashed line is a Gaussian fit of the measured resonance length shifts (1.8- $\mu \mathrm{m}$ waist). The change in linewidth is reported in (b). (c) Similar measurement realized while scanning the nanowire along the optical axis, revealing the standing-wave structure of the cavity mode. (d) Transmission and scatter maps measured when scanning the nanowire in the horizontal $X Z$ plane while locking the cavity on resonance. The cavity-length shift map is obtained via the correction of the feedback loop, after substraction of the slow experimental drift. (e) Similar measurements obtained when scanning the nanowire in the vertical $Y Z$ plane $(X=0)$. The optical axis is marked with a dashed line. The parametric coupling strength $G_{z}$ is obtained by spatial derivation of the cavity shift map along $\mathbf{e}_{\mathbf{z}}$.

The maximum slopes observed amount to $G_{x} / 2 \pi \approx$ $0.3 \mathrm{GHz} / \mathrm{nm}$ and $G_{z} / 2 \pi \approx 3 \mathrm{GHz} / \mathrm{nm}$. Those measurements were realized with a nanowire featuring zero-point fluctuations spreading over $\delta r_{\mathrm{zpf}}=\sqrt{\hbar / 2 M_{\mathrm{eff}} \Omega_{\mathrm{m}}} \approx 0.4 \mathrm{pm}$, so the single-photon vectorial coupling strength $\left(\mathbf{g}_{\mathbf{0}} \equiv\right.$ $\mathbf{G} \delta r_{\text {zpf }}$ ) is as large as $g_{0}^{z} / 2 \pi=1.2 \mathrm{MHz}$. This value, already rather large compared to other implementations [1], is significantly larger than the nanowire fundamental frequency $\left(\Omega_{\mathrm{m}} / 2 \pi=50 \mathrm{kHz}\right.$ here $)$, thus largely entering the singlephoton regime of the parametric interaction $\left(g_{0} / \Omega_{\mathrm{m}}=25\right)$. The maximum linewidth broadening caused by the nanowire thermal noise [36], spreading over $\Delta r^{\text {th }}=\sqrt{k_{B} T / M_{\mathrm{eff}} \Omega_{\mathrm{m}}^{2}} \approx$ $7 \mathrm{~nm}$, amounts to $G_{z} \Delta r^{\text {th }} \approx 2 \pi \times 23 \mathrm{GHz}$ at $300 \mathrm{~K}$, which becomes comparable to the broadened optical resonance [see Fig. 2(b)] but remains small compared to the observed parametric shift $(400 \mathrm{GHz})$.

\section{NANOWIRE-BASED CHARACTERIZATION OF THE INTRACAVITY FIELD}

In view of its subwavelength-sized diameter, the nanowire can be used to map the intracavity mode structure.
Because of the finite-laser mode-hop-free tunability (approximately $30 \mathrm{GHz}$ ), we choose to lock the cavity on the laser wavelength and thus compensate the nanowireinduced optical resonance shift with the cavity piezos. The error signal is synthesized from the cavity transmission signal using a $250-\mathrm{kHz}$ lock-in. We use a dual feedback loop acting on the fast and slow piezo elements with a bandwidth intentionally restricted to a few $\mathrm{kHz}$ in order not to compensate for resonant mechanical vibrations of the nanowire. Figures 2(d) and 2(e) represent the experimental transmission, scatter (laterally collected through the objective), and correction signals recorded when scanning the nanowire in the horizontal $X Z$ (vertical $Y Z$ ) plane. These measurements permit a direct visualization of the intracavity standing wave, as revealed in the transmission maps. The intracavity nodes (antinodes) appear as regions of large (low) transmission.

The scatter maps reflect a different behavior. Progressively inserting the nanowire in the cavity mode, closer to an antinode, first causes an increase of the scattered light, as expected. However, this increase is followed by a reduction (white central areas), which simply originates from the 
reduction of the cavity finesse due to the increased loss rate. This transition from a nanowire scattering rate that is smaller or larger than the bare cavity loss rate is responsible for the ring shapes observed in Fig. 2(d). The same considerations also explain the signatures observed on the vertical maps, where the dispersive and dissipative optomechanical coupling rates can be tuned by adjusting the vertical insertion of the nanowire extremity in the cavity mode. As long as the nanowire stays out of a scatter ring, the cavity finesse remains almost unchanged. Interestingly, positions on each side (along $\mathbf{e}_{\mathbf{z}}$ ) of the optical nodes where the cavity finesse remains almost unchanged also feature large parametric coupling strength, as shown in Fig. 2(e) (panel $G_{z}$ ), and are where the largest optomechanical backaction can be expected.

Those multichannel scanning probe maps are impacted by several parameters, such as the nanowire diameter, laser wavelength and polarization, cavity geometry (curvature, length, alignment), and finesse. They can all be tested quantitatively through this approach, which thus presents a unique opportunity to investigate the intracavity field in confined microcavities.

\section{NANO-OPTOMECHANICAL INVESTIGATION OF THE INTRACAVITY FORCE}

The above measurements allowed us to investigate how the oscillator perturbs the intracavity field, which represents one facet of the optomechanical interaction. To fully quantify an optomechanical system, it is essential to characterize the second facet of the interaction and measure the action of the intracavity light field on the oscillator. This dual characterization is essential, in particular for the "oscillator in the middle" approaches, when the nature and excitation level of the optical mode involved in the interaction Hamiltonian (described by the $\hat{a}, \hat{a}^{\dagger}$ operators) strongly depends on the oscillator position (optical mode spatial profile, oscillator induced losses,...) and on the pumping conditions. We show here that the large force sensitivity of the nanowire, combined with the large coupling strength achieved thanks to the small-modevolume microcavity mode allows us to detect optical forces corresponding to modulations of the mean intracavity photon number smaller than unity.

We measure the intracavity force field using a pumpprobe scheme [48] realized by modulating the intracavity intensity at frequencies close to the mechanical resonances while recording the laser-driven nanowire trajectories with a separate probe laser.

The visible probe laser (few $\mu \mathrm{W}$ at $633 \mathrm{~nm}$ falling outside of the microcavity coating reflection window) is coinjected in the cavity fibers. The reflected power map $P_{R}(\mathbf{r})$, which is recorded on a separate photodiode [see Fig. 1(d) and Appendix A], presents an interference pattern (between reflections on the nanowire and input fiber extremity) that strongly depends on the nanowire position $\mathbf{r}=\mathbf{r}_{\mathbf{0}}+\delta \mathbf{r}$. The vibrations of its extremity $\delta \mathbf{r}(t)$ are dynamically encoded as photocurrent fluctuations $\delta P_{R}(t)=$ $\left.\delta \mathbf{r}(t) \cdot \nabla P_{R}\right|_{\mathbf{r}_{0}}$, which allows us to measure the nanowire vibrations $\delta r_{\beta}(t)=\delta \mathbf{r}(t) \cdot \mathbf{e}_{\beta}$ projected along the local measurement vector $\mathbf{e}_{\beta} \equiv \nabla P_{R} /\left|\nabla P_{R}\right|$. Following the readout protocols described in Refs. [48,50,52], we can probe the nanowire thermal noise [see Fig. 3(a), grey curve] to determine its effective mass and verify its proper thermalization, but also its response to an external force such as the one exerted by the intracavity light field. During those measurements, the cavity is locked on the pump laser, whose intensity is partially time modulated using an acousto-optic modulator: $P_{0}+\delta P \cos \Omega t$, with an amplitude $\delta P$ around a mean value $P_{0}$. The modulation frequency $\Omega / 2 \pi$ is swept across both eigenmode frequencies using a vectorial network analyzer [48], and we record the optically induced driven displacement $\delta r_{\beta}[\Omega]$ as shown in Fig. 3(a) (black dots). The individual responses of each transverse mechanical mode are well distinguishable. The responses are well fitted in amplitude and phase with the complex expression

$$
\delta r_{\beta}[\Omega]=\mathbf{e}_{\beta} \cdot \sum_{i=1,2} \chi_{i}[\Omega]\left(\mathbf{e}_{\mathbf{i}} \cdot \delta \mathbf{F}\right) \mathbf{e}_{\mathbf{i}}
$$

where $\chi_{i}^{-1}[\Omega] \equiv M_{\mathrm{eff}}\left(\Omega_{i}^{2}-\Omega^{2}-i \Omega \Gamma\right)(i=1,2)$ are the inverse mechanical susceptibilities of each eigenmode. The measurement vector $\mathbf{e}_{\beta}$ is independently determined using the standard calibration protocols $[48,50]$. The eigenmode orientations, frequencies, damping rates, and effective masses are independently determined by fitting the Brownian motion spectra in the absence of light modulation (see Appendixes A and B). Mode 1 is pointing at $+20^{\circ}$ from the optical axis $\mathbf{e}_{\mathbf{z}}$. As such, the only fitting parameter for the response measurement is the modulated force complex vector $\delta \mathbf{F}$ caused by the intensity modulation $\delta P$ of the input light, which is modulating the mean intracavity photon number. Since the experiment is realized in the adiabatic cavity regime $\left(\Omega_{\mathrm{m}} \ll \kappa\right)$, pure optical forces instantaneously follow (on mechanical timescales) the input light modulation, while photothermal forces [48] will be in quadrature (see Appendix B). The amplitudes and orientations of the instantaneous optical force are reported in Figs. 3(b) and 3(c) for increasing modulation depth of the intracavity photon number (produced by a modulation $\delta P$ of the pump intensity). Thus, we can verify the linearity of the system response and demonstrate its sensitivity to variations of the mean intracavity photon number smaller than unity. We measure a transduction efficiency of approximately $340 \mathrm{aN} /$ photon for the optical force per intracavity-photon-number change (or a $2 \mathrm{fN} / \mu \mathrm{W}$ transduction efficiency related to the input optical power) at a given position of the nanowire extremity in the optical mode [indicated by $\odot$ in Figs. 2(d) and 2(e), slightly beside a node]. As expected, the optical force is aligned with the 

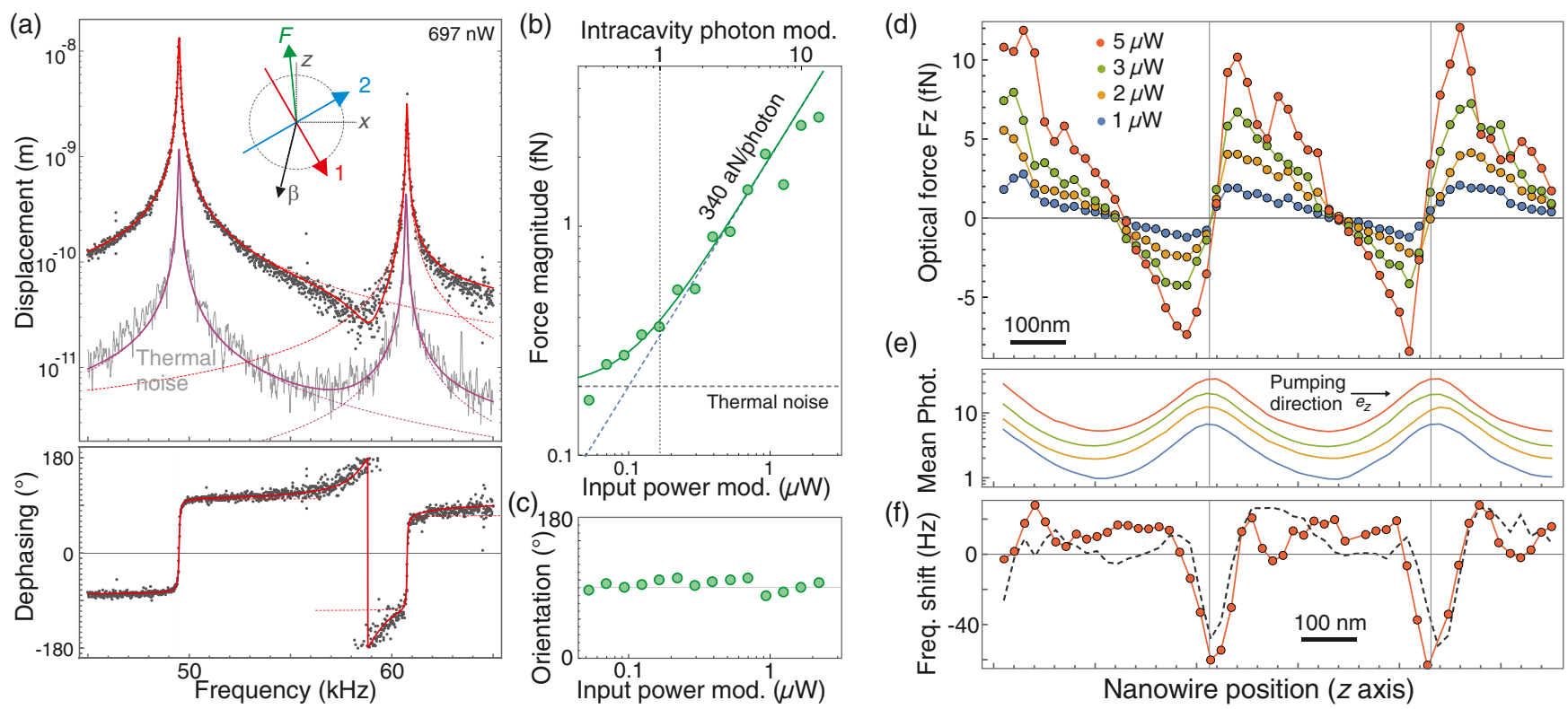

FIG. 3. Nanomechanical measurement of the intracavity optical force. (a) Thermal noise (gray line, 30-Hz resolution bandwidth) and response measurements (dots) measured with the red probe laser $(5 \mu \mathrm{W})$, providing a projective measurement of the nanowire vibrations along the $\mathbf{e}_{\beta}$ axis (inset), for a cavity locked at resonance at position $\odot$ in Figs. 2(d) and 2(e). The response measurements are realized with a vectorial network analyzer, whose output channel serves to intensity modulate the infrared cavity pump laser. The phase difference is measured between the recorded displacement and the generated intensity modulation. Fitting of the data (see text) allows us to determine the eigenmode orientations $\mathbf{e}_{\mathbf{1 , 2}}$ and the instantaneous optical force vector. (b,c) Evolution of the magnitude and orientation (with respect to $\mathbf{e}_{\mathbf{x}}$ ) of the optical force vector plotted with respect to the amplitude of the intracavity-photon-number modulation $\left(\delta\left\langle a^{\dagger} a\right\rangle\right.$ ), down to unity, and to the corresponding input power modulation amplitude (using $P_{0}=3 \mu \mathrm{W}$, which corresponds to 17 intracavity photons at that position; see Appendix D). (d) Dependence of the instantaneous force $F_{z}$ with the position along the optical axis for increasing input powers (70\% modulation depth). The nodes of the electromagnetic modes are indicated as vertical lines and correspond to maxima in the transmission plots shown in (e), expressed in terms of mean intracavity photon number. (f) Measured relative frequency shifts (dashed lines) obtained for $5 \mu \mathrm{W}$ compared to the shifts expected from the measured optical-force-field gradients $\left(-1 / 2 \Omega_{\mathrm{m}} M_{\mathrm{eff}}\right) \partial_{z} F_{z}$ (red dots).

optical axis $\left(\mathbf{e}_{\mathbf{z}}\right)$, as reported in the inset of Fig. 3(a). The magnitude of the measured force per intracavity photon is in good agreement with numerical simulations (see Appendix D and Fig. 18), predicting a $260 \mathrm{aN} /$ photon transduction efficiency. At this position, the nanowire thermal noise limits its sensitivity to variations of the mean intracavity photon number at the level of 0.07 photon $/ \sqrt{\mathrm{Hz}}$ at $300 \mathrm{~K}$.

Identical response measurements were subsequently realized at various locations along the optical axis. The resulting instantaneous optical force is reported in Fig. 3(d). It was measured with a fixed modulation depth $\delta P / P_{0} \approx$ 0.7 for increasing average input powers $P_{0}$ (from 1 to $5 \mu \mathrm{W})$. The measured optical force is $\lambda / 2$ periodic due to the standing-wave periodicity. This type of force measurement inside a cavity is original. We found that it presents a repulsive character in the vicinity of the nodes of the intracavity field and, on the contrary, is found to be weakly attractive towards the antinodes of the intracavity field. Because of the multipolar character of the nanowire optical response, the interpretation of the force map can be delicate. However, the phenomenology is well explained by theoretical modeling (see Appendix C) and can be understood by the following qualitative argument, which also holds for membrane-in-the-middle experiments [38], for which the optical mode can be split into two subcavities located on each side of the oscillator. Regions of positive (resp. negative) dispersive shifts, $G_{z}>0(<0)$, are then associated with situations where a larger amount of light is stored in the second (resp. first) subcavity, so the total force is found to be negative (resp. positive). Also, the approximately $30 \%$ positive-negative asymmetry observed in the force extrema is connected to the laser pumping direction (along $\mathbf{e}_{\mathbf{z}}$ ) and mirror asymmetries. The "sawtooth" pattern visible in the force variation along the optical axis is a consequence of the finesse reduction observed in the antinode areas. There, the force deviates from the oscillatory pattern that is expected from the parametric measurement of $G_{z}$ shown in Fig. 2.

The intracavity force field exerted on the nanowire $\mathbf{F}(z)$ presents some large spatial variations of $\partial_{z} F_{z} \approx 1.5 \times$ $10^{-7} \mathrm{~N} / \mathrm{m}$ for $5 \mu \mathrm{W}$ input power in the vicinity of the intracavity nodes. They add up to the intrinsic nanowire stiffness, $k=M_{\mathrm{eff}} \Omega_{\mathrm{m}}^{2} \approx 10^{-4} \mathrm{~N} / \mathrm{m}$, and are responsible for 


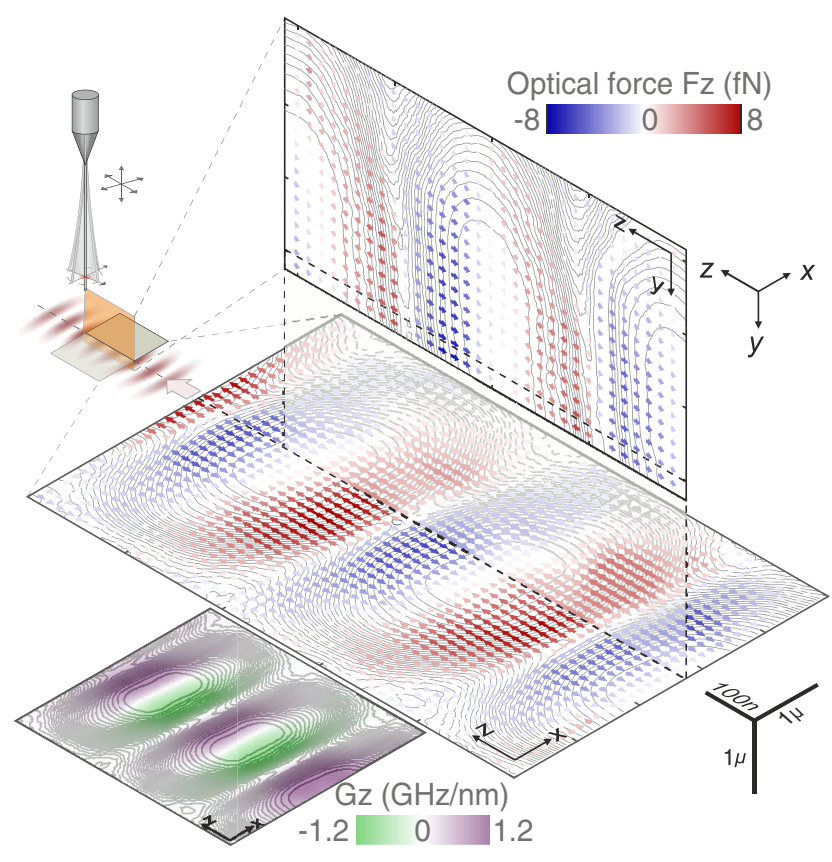

FIG. 4. Nano-optomechanical mapping of the intracavity force field. We show horizontal $(X Z)$ and vertical $(Y Z)$ maps of the intracavity force field (anisotropic length scales), measured for an injected power of $3 \mu \mathrm{W}$ at $767 \mathrm{~nm}$. The pump laser is injected along $\mathbf{e}_{\mathbf{z}}$, and the dashed line represents the optical axis. The color code indicates the force component along the optical axis $F_{z}$. Inset: parametric coupling strength $G_{z}$ derived from the correction of the cavity lock. The transmission isovalues are superimposed as gray lines to help visualize the intracavity field structure.

frequency softening at the node locations. The measured frequency shifts are shown in Fig. 3(f) and are in good agreement with the ones deduced from the gradient of force measurements, which validates the force measurement protocol exposed above. We point out that those force measurements were realized using a largely frequency-split nanowire (approximately 20\%), in order not to experience eigenmode rotations, which would have otherwise required a vectorial 2D readout of the nanowire displacements [50,52].

We pursue those measurements by mapping the intracavity $2 \mathrm{D}$ force field in $3 \mathrm{D}$. Here, we employ a faster measurement scheme in order not to suffer from spatial drifts, which should remain smaller than $10 \mathrm{~nm}$ over a few hours (corresponding to a $0.01^{\circ}$ temperature stability). Instead of a full response measurement [Fig. 3(a)], we realize a simultaneous multifrequency excitation drive at each position. A triplet of three optical drive tones is employed per mechanical mode, separated by $50 \mathrm{~Hz}$, whose central frequency is locked onto the nanowire eigenfrequency with a soft-peak tracking loop (see Appendix B 4). Thus, we can simultaneously determine the local force, the mechanical frequencies, and quality factors while reducing the measurement time per point to $100 \mathrm{~ms}, 100$ times faster than full response measurements. The measurement vector $\mathbf{e}_{\beta}$ is also locally recorded in real time using a two-tone $(80-85 \mathrm{~Hz})$ lock-in detection scheme (see Appendix B 4 and Fig. 12).

The resulting $X Z$ and $Y Z$ maps of the intracavity force field are shown in Fig. 4, superimposed on the transmission maps encoded as isovalues to help localize the intracavity field structure. The color code shows the measured optical force projected along the optical axis $\left(F_{z}\right)$, shown as colored arrows in the maps. Those measurements are realized using $400 \times 400$ pixels, lasting over 4 hours, and the signal processing routine described in Appendix B 4 employs signal averaging up to 10 neighboring pixels, which correspond to a spatial resolution of $25 \mathrm{~nm}$ along the $z$ axis. One can recognize the antitrapping or trapping structures in the vicinity of the nodes or antinodes, and the vanishing of the optical force when the nanowire is extracted out of the intracavity mode volume, both transversally $(x)$ and vertically $(y)$. The parametric coupling map $\left(G_{z}\right)$ is derived by calculating the gradient of the cavity-lock correction signal map [see Fig. 2(d), "cavity shift" panel], recorded during the same $X Z$ measurement sequence. On each side of the antinodes, it is possible to identify locations of simultaneous, large optical backaction and large parametric coupling strength. Those regions are clearly of great interest for future experiments in the single-photon regime.

We note that operating with a quasifrequency-degenerated nanowire and a full 2D readout scheme will allow future investigations of the possible nonreciprocal (nonconservative [52]) character of the intracavity force field and its impact on the nanowire dynamics.

\section{CONCLUSION AND PROSPECTIVES}

We have realized a dual characterization of the optomechanical interaction, via nanowire-mediated scanning probe measurements of the intracavity field and mapping of the intracavity optomechanical force field using intracavityphoton-number modulations smaller than unity. This method permits us to explore confined optical fields and provides a novel exploration tool, complementary to purely optical scanning probe measurements. The use of nanowires with subwavelength-sized diameters permits us to operate with ultrasensitive force sensors, but as a counterpart, it generates a large optical scattering. However, this does not prevent us from largely entering the single-photon regime at the condition to properly map the optomechanical interaction and to operate in the vicinity of the intracavity field nodes.

For consistency, all the experiments described in the paper were obtained with the same nanowire, but larger coupling strengths, up to $g_{0}^{z} / 2 \pi=2.6 \mathrm{MHz} \approx 100 \times \Omega_{\mathrm{m}} / 2 \pi$, were reached with different nanowires (see Fig. 8). We note that the light-nanowire interaction can be further engineered and enhanced by exploiting higher-order internal optical resonances (Mie) of the nanowire [57,58], benefiting from their multipolar optical response. Since we operate in the adiabatic regime, many perspectives of this work are found in quantum 
optics, where the large optomechanical interaction serves as a nonlinear resource operating down to the single intracavity photon level. In particular, it will allow us to investigate the deviations from the semiclassical approximation-where the fluctuations of the intracavity field have a similar impact as the mean field - and reaching a static bistability regime for less than one intracavity photon. To do so, one needs to reduce the oscillator thermal noise down to a level where the singlephoton recoil $\delta x^{(1)}$ becomes visible.

Operating with ultrasensitive $\mathrm{kHz}$ nanowires (see Table I, NW3), such as the ones developed for operations at dilution temperatures [60], should permit us to reach approximately 7-MHz single-photon coupling strength while reducing their thermal noise down to $\sqrt{n_{T}} \approx 500$ times their zero-point fluctuations when thermalized at $20 \mathrm{mK}$. A single intracavity photon should then produce a static deformation of $\delta x^{(1)} \approx 8000 \delta x_{\text {zpf }}$, which would be largely detectable on top of the nanowire residual rms thermal noise.

Furthermore, the exotic regime of single-photon bistability should be achievable in our system since large single-photon parametric cooperativities $\mathcal{C}^{(1)} \equiv 2 g_{0}^{2} / \Omega_{\mathrm{m}} \kappa \approx$ 10 can be achieved even with a modest cavity linewidth (6 GHz). We also note that single-photon cooperativities [1] as large as $g_{0}^{2} / \Gamma_{\mathrm{m}} \kappa \approx 4.2 \times 10^{5}$ are within reach.

Finally, one can estimate the possibility to detect the quantum fluctuations of the radiation-pressure force noise in the single-photon regime, which become observable if they exceed the Langevin force fluctuations. For a single, mean intracavity photon (see Appendix E), the ratio of the spectral densities of their respective force noise can be expressed [5] as $2 C^{(1)} Q / n_{\mathrm{T}}$, which could reach approximately 30 at dilution temperatures.

Those considerations are thus strong incentives for pushing the developments towards dilution temperatures to explore, with mesoscopic oscillators, the regime of single-photon cavity optomechanics and possibly open the road towards scanning probe measurements of vacuum Casimir forces within confined optical resonators.

\section{ACKNOWLEDGMENTS}

We warmly thank the PNEC group at ILM; T. Heldt for his assistance in the early phase of the experiment; as well as S. Reynaud, F. Pistolesi, J. Estève, J. P. Poizat, G. Bachelier, J. Jarreau, C. Hoarau, E. Eyraud, A. Laurent, and D. Lepoittevin. F. F. acknowledges funding from the Laboratoire d'Alliances Nanoscience-Energies du Futur (ANR-10-LABX-51-01). P. H. acknowledges funding from the European Union H2020 programme (Marie Sklodowska-Curie Grant No. 754303) and QUENG. This project is supported by the French National Research Agency [projects QCForce (ANR-16 CE090028) SinPhoCOM (ANR-19-CE47-0012) LANEF framework (ANR-10-LABX-51-01)(CryOptics), Investissement d'avenir program (ANR-15-IDEX-02) (CARTOF and
QUENG)] and by the European Research Council under the EU's Horizon 2020 Research and Innovation Programme, Grant Agreements No. 671133 (EQUEMI project), No. 767579 (CARTOFF), and No. 820033 (AttoZepto). J. R. acknowledges support by the Paris Ilede-France Region in the framework of DIM SIRTEQ.

\section{APPENDIX A: EXPERIMENT}

\section{Description of the experiment}

A complete scheme of the experimental setup is given Fig. 5. The infrared laser source is a tunable and low-noise Ti:sapphire laser, with sub-100-kHz linewidth. It is employed between 760 and $820 \mathrm{~nm}$ depending on the optical finesse needed. The finesse can thus be tuned between 200 and 45000 . Its output is spatially filtered using a monomode fiber, and its intensity is controlled with an acousto-optic modulator. A nonpolarizing 90:10 beam splitter is employed to collect the light reflected from the cavity, and the injection polarization is controlled by a $\lambda / 2$ wave plate. The experiment is operated in a vacuum chamber to eliminate acoustic damping. A pressure below $10^{-2}$ mbar can be statically maintained over several days, which is sufficient to realize the long-term acquisition needed. The vacuum chamber is suspended above an optical table using four bungee cables to reduce acoustic vibrations, and it is inserted in a thermal and acoustic foam insulation chamber to improve its temperature stability and further reduce acoustic vibrations.

The injection fiber is directly attached to a breadboard in the vacuum chamber, while the transmission fiber is mounted on a motorized $X Y Z$ stage and on a gimbal mount to adjust the fiber lateral position and its orientation with respect to the injection fiber. Both fibers are supported by annular piezo elements, which are used to control and dither the cavity length. A coarse piezo stack enables an approximately $1.1-\mu \mathrm{m}$ scan range, which is sufficient to cover several free spectral ranges of the cavity. A rapid piezo element is employed to dither the cavity length by approximately $0.1 \mathrm{~nm}$ at $250 \mathrm{kHz}$, in order to provide the error signal for the cavity lock.

The cavity transmission is detected outside of the optical chamber, while a 20x/0.4NA microscope objective, positioned perpendicularly to the optical axis, serves to collect the light scattered by the nanowire. The later is focused on a photodiode positioned on the vacuum chamber support.

The nanowire is mounted on a $X Y Z$ piezo stage, providing travel ranges of $30 \times 30 \times 10 \mu \mathrm{m}$ along the $X Y Z$ axes. It is supported by a motorized $X Y Z$ stage used for coarse positioning. The nanowire support can be rotated in order to align the nanowire with the $X Y$ plane, which allows us to insert the nanowire in small-mode-volume optical modes. In practice, we first optimize the second fiber angles in the absence of nanowire; we then open the cavity up to a length of $200 \mu \mathrm{m}$, insert the nanowire in the 


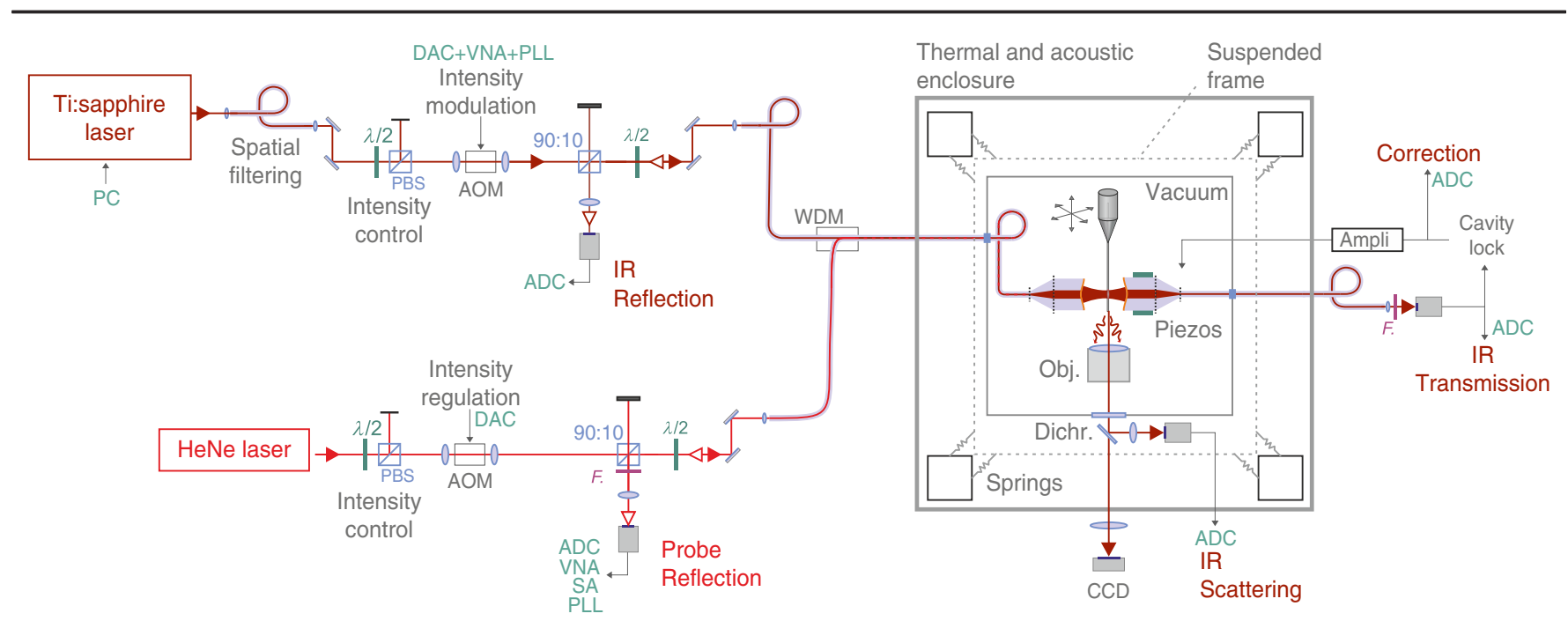

FIG. 5. Detailed experimental setup. (F.: filters, ADC/DAC: inputs/outputs of the acquisition card; Dichr.: dichroic filter; WDM: wavelength combiner; IR: infrared.) The core experiment is realized in a vacuum chamber, mounted on a suspended plate, which hosts the lateral IR scatter detector. The experiment is mounted in a multilayer acoustic and thermal isolation box.

mode volume area, and progressively close the cavity while continuously optimizing the angles and lateral position of the second fiber. All of these prealignment steps can be realized by monitoring the transmission signals.

Since the optical shifts induced by the nanowire are extremely large $(400 \mathrm{GHz})$, significantly larger than the mode-hope free scan range of the laser $(30 \mathrm{GHz})$, we choose to lock the cavity length on the laser frequency. The error signal and the feedback gains are produced and processed by a Field Programmable Gate Arrays card based on the PyRPL [61] code developments. We dither the cavity length using the rapid piezo element and give feedback on the piezo stack at low frequencies (up to approximately $200 \mathrm{~Hz}$ ), while a second loop employs the fast piezo (up to a few $\mathrm{kHz}$ ). The lock is rather robust and agile, and allows us to maintain the cavity at resonance for days, even in the presence of the nanowire, which can largely reduce the transmission level (down to 5\%). The lock bandwidth is intentionally limited in order not to compensate for the dynamical parametric cavity shifts induced by the nanowire motion. As such, mechanical motion remains visible in the lock error signal, which provides a complementary readout channel for mechanical vibrations. Depending on the experiments envisioned, one can also increase the lock bandwidth beyond the nanowire mechanical resonance (the first internal resonance of the fast piezo ring element is around $300 \mathrm{kHz}$ ) in order to compensate for position fluctuations so that the cavity remains dynamically locked.

The mirror curvatures are measured during the microfabrication phase. The determination of the cavity length is realized by measuring the spectral distance between two optical resonances using the large tunability of the pump lasers and by measuring the spectral arrangement of the transverse mechanical modes. Measurements were realized with two sets of almost identical fibers, with radii of curvature $29 \mu \mathrm{m}$ and $60 \mu \mathrm{m}$. All of the measurements shown in this article are obtained with the first set of fibers. We have also investigated the parametric coupling and the force-field structure of other transverse modes, as well as cross-couplings induced by the nanowire. The nanowires allow for a clear identification of the nature intracavity modes. Also, we employ the nanowire in order to carefully align the intracavity mode: Depending on the fiber positions and orientations, the optical mode can be twisted with respect to the fiber axes.

In order to probe the nanowire vibrations independently from the cavity mode, we make use of a visible 633-nm laser, which is coinjected in the cavity fibers using fibered wavelength dividing modules (WDM) and whose reflection is collected using a 90:10 nonpolarizing beam splitter. The partial reflections on the fiber extremity (falling outside of the

TABLE I. Nanowires discussed in this work. NW1 and NW2 were tested at room temperature in the cavity. NW3 is an estimation based on the typical nanowires investigated at dilution temperatures [60] with a $10-\mu \mathrm{m}$ cavity length.

\begin{tabular}{lcccccccr}
\hline \hline NW & $\mathrm{L}(\mu \mathrm{m})$ & $\mathrm{d}(\mathrm{nm})$ & $\Omega_{\mathrm{m}} / 2 \pi(\mathrm{kHz})$ & $\mathrm{Q}$ & $\mathrm{M}_{\mathrm{eff}}(\mathrm{pg})$ & $\mathrm{dF}_{\min }\left(\mathrm{aN} / \mathrm{Hz}^{1 / 2}\right)$ & $\mathrm{g}_{0}^{z} / 2 \pi(\mathrm{MHz})$ & $g_{0} / \Omega_{\mathrm{m}}$ \\
\hline NW1 (article) & 70 & 130 & $50-60$ & 2000 & 0.7 & $25(300 \mathrm{~K})$ & 1.3 & 25 \\
NW2 (SI Fig. 8) & 90 & 160 & $27.2-27.6$ & 2700 & 2 & $30(300 \mathrm{~K})$ & 2.6 & 96 \\
NW3 & 400 & 160 & 1.6 & 100000 & 6.6 & $0.02(20 \mathrm{mK})$ & 7 & 4300 \\
\hline \hline
\end{tabular}


reflectivity window of the mirror coatings) and the one coming from the nanowire interfere and are responsible for a spatial $X Z$ structuration of the red reflection image. When the nanowire oscillates, it dynamically modulates the reflected signal, and the principles of projective readout $[48,50]$ can be similarly employed (see Fig. 12). The experiment is interfaced by software that controls the nanowire position in 3D and the cavity length, and records the different optical signals, as well as demodulation signals produced by the phase locked loop (see below). The nanowire vibrations are recorded using signal and network analyzers and a dual phase locked loop that is employed for the multifrequency response measurements (see below).

The experiment is prealigned at room pressure by taking special care on the fiber tilt angles in order to produce an optical cavity mode presenting a good mode matching to the fibers, oriented perpendicularly to the nanowire axis (and, if possible, along the $Z$ axis of the piezo stage moving the nanowire). Before pumping the chamber, we retract the fibers by approximately $100 \mu \mathrm{m}$ so that the pump vibrations do not make the nanowire stick to the fiber end faces, and the system is realigned at low pressure. It is important to stop the side illumination during the fine alignment phase, in order to limit spatial drifts in the experiment. On some occasions, we observe electrostatic charging in the system, which causes the nanowire to stick to the fiber extremities. In general, after a few hours, the effect disappears. The fiber sides are metal coated to sustain the optical coating, which also serves to partly shield the fiber extremities from the stray electric field produced by the closest piezo elements $(0-100 \mathrm{~V}$, at $1-\mathrm{cm}$ distance). Their impact on the nanowire dynamics - the nanowire is attracted to regions of large electric field - could occasionally be detected for very small cavities, as a residual forcefield gradient, but, most of the time, are far smaller than the optical forces involved in the experiment.

\section{Internal Mie resonances}

Figure 6 illustrates the role of internal Mie resonances on the light-nanowire interaction.

\section{Nanowire samples}

Several nanowires are employed in this project (see Table I). For a given cavity mode, the parametric coupling strength $(\vec{G})$ only depends on the nanowire diameter. We employ different nanowires with diameters ranging from 100 to $250 \mathrm{~nm}$. All the results presented in the article are realized with a 130-nm-diameter nanowire, while the largest coupling strengths could be achieved using a 160-nm-diameter nanowire, as shown in Fig. 8.

The NW3 line represents an extrapolation at dilution temperatures based on recent results obtained [60], where we could detect the thermal noise of $\mathrm{SiC}$ nanowires thermalized down to $20 \mathrm{mK}$ using a single-path optical readout. Using a modest $6-\mathrm{GHz}$ cavity linewidth (2000
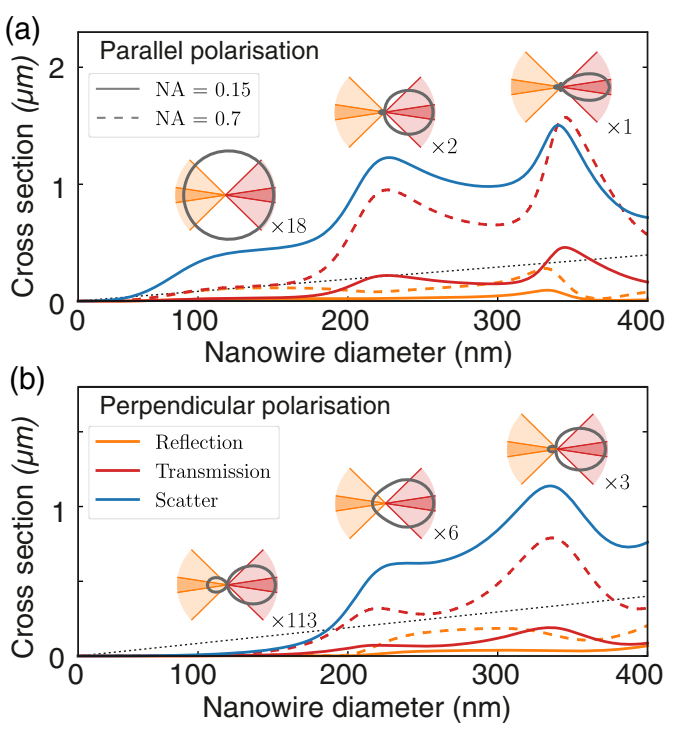

FIG. 6. Nanowire multipolar optical response: Mie resonances. Scattering cross sections for infinite $\mathrm{SiC}$ cylinders under planewave illuminations incoming from the left-hand side, for increasing nanowire diameters. The reflection, transmission, and scatter components are integrated in the backward, forward, and lateral angular segments, respectively. The total linear cross section can largely exceed the nanowire diameter (indicated by a black line) and varies rapidly with the nanowire diameter due to the Mie resonances. The nanowire optical response can be assimilated to a dipole for diameters smaller than approximately $100 \mathrm{~nm}$, while its multipolar character must be taken into account for the nanowires employed in that work (130 and $160 \mathrm{~nm}$ in diameter).

finesse), this nanowire should permit us to reach a singlephoton parametric cooperativity of $\mathcal{C}^{(1)} \equiv 2 g_{0}^{2} / \Omega_{\mathrm{m}} \kappa=9.5$ and a single-photon cooperativity of $g_{0}^{2} / \Gamma_{\mathrm{m}} \kappa=426000$.

\section{Aspect ratio of the scanning images}

Figure 7 illustrates aspects ratio considerations of the scanning images.

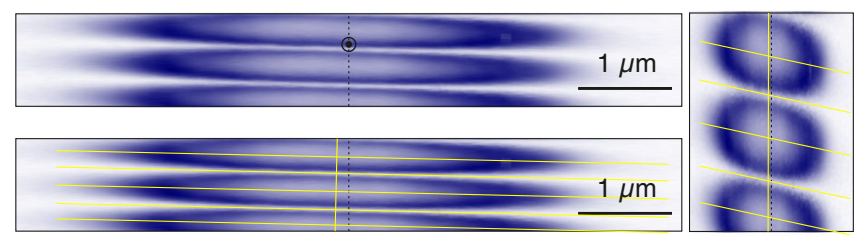

FIG. 7. Aspect ratio of the scanning images. Representations of the Fig. 2(d) scatter channel are shown with their true aspect ratio on the left. In the lower-left plot, a tilted perpendicular grid has been added. The tilt angle is $-1.7^{\circ}$. The black circle represents the dimensions of a 150-nm-diameter nanowire, given for comparison. The image axes $(X Z)$ are the ones set by the $X Y Z$ piezo stage moving the nanowire. Right: the same image shown with the squeezed aspect ratio employed in Fig. 2 on the right. The deformation of the perpendicular grid is an artefact of the aspect ratio chosen. 

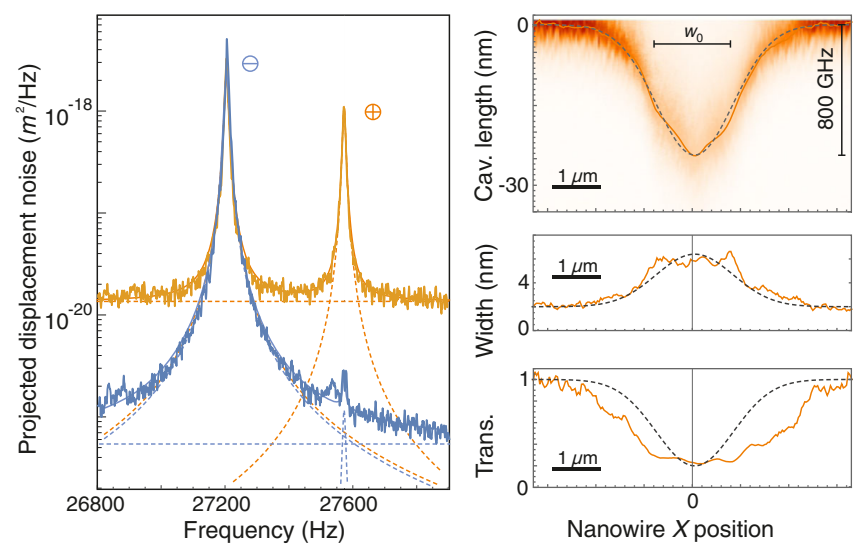

FIG. 8. Coupling strength with a 160-nm-diameter nanowire (NW2). Left: thermal noise at $300 \mathrm{~K}$ of a 160 -nm-diameter nanowire, measured along two measurement channels [50]. It presents an effective mass of $2 \times 10^{-15} \mathrm{~kg}$, oscillates at 27200 and $27580 \mathrm{~Hz}$, with a $\Gamma_{\mathrm{m}} / 2 \pi=10 \mathrm{~Hz}$ damping rate. In this measurement, the lower eigenmode orientation is oriented perpendicular to the optical axis, to be ideally read by the differential channel $\ominus$. It conveys a $30 \mathrm{aN} / \sqrt{\mathrm{Hz}}$ force sensitivity, with thermal noise spreading over $\Delta x_{\mathrm{th}}=7.8 \mathrm{~nm}(64 \mathrm{pm})$ at $300 \mathrm{~K}(20 \mathrm{mK})$ and zero-point fluctuations spreading over $\delta x_{\mathrm{zpf}}=0.4 \mathrm{pm}$. Right: cavity resonant length shifts measured at $767 \mathrm{~nm}$ while scanning the nanowire transversally ( $x$ axis) across the cavity mode, through an antinode (fitted optical waist of $w_{0}=1.6 \mu \mathrm{m}$ ). The maximum parametric shifts amount to a cavity length change of $-24.7 \mathrm{~nm}$ (total length $12 \mu \mathrm{m}$ ), corresponding to an equivalent $+800-\mathrm{GHz}$ resonance frequency increase. The corresponding maximum slopes are $G_{x} / 2 \pi=$ $0.6 \mathrm{GHz} / \mathrm{nm}$ and $G_{z} / 2 \pi=6.5 \mathrm{GHz} / \mathrm{nm}$, and they lead to a single-photon coupling strength of $\mathbf{g}_{\mathbf{0}}=(0.24,2.6) \mathrm{MHz}$, which represents a maximum ultrastrong coupling ratio $g_{0} / \Omega_{\mathrm{m}}$ of approximately 100 . The dynamical $\mathrm{rms}$ broadening of the cavity resonance is approximately $G_{z} \Delta x_{\text {th }} \approx 2 \pi 50 \mathrm{GHz}$ in frequency, or $1.6 \mathrm{~nm}$ in effective resonance width, which is slightly smaller than the 4-nm line broadening observed [36].

\section{Optomechanical coupling to nanowire NW2}

Figure 8 illustrates the coupling strength obtained with a 160-nm-diameter nanowire.

\section{APPENDIX B: FORCE MEASUREMENTS}

The optical force measurements are realized using a pump-probe technique. The nanowire vibrations are read out using the red probe beam, while the optical force is intensity modulated around a mean static value.

\section{Optical readout of the nanowire vibrations}

Because of the interference between the probe light reflected from the input cavity mirror and the one reflected from the nanowire and collected back into the fiber, the reflected intensity $\left[P_{R}(\mathbf{r})\right]$ presents spatial variations that depend on the nanowire position. When the nanowire oscillates with a vectorial deflection $\delta \mathbf{r}$ around its rest position $\mathbf{r}_{\mathbf{0}}$, it generates a modulated output voltage of $P_{R}\left(\mathbf{r}_{\mathbf{0}}+\delta \mathbf{r}(t)\right) \approx P_{R}\left(\mathbf{r}_{\mathbf{0}}\right)+\left.\delta \mathbf{r}(t) \cdot \boldsymbol{\nabla} P_{R}\right|_{\mathbf{r}_{0}}$. The fluctuations of the photodiode output let us realize a projective readout of the nanowire motion $\delta \mathbf{r}$ along a measurement vector $\mathbf{e}_{\beta}$, which is defined as $\left.\mathbf{e}_{\beta} \equiv \boldsymbol{\nabla} P_{R}\right|_{\mathbf{r}_{0}} /\left|\boldsymbol{\nabla} P_{R}\right|_{\mathbf{r}_{0}} \mid$. The latter is determined by measuring the local gradient of the reflected power map. To do so, we employ two methods: a static one and a dynamical one. In the static mode, we record the reflected signal for different positions of the nanowire, placed on a 20-nm grid around the measurement position. We then compute the local tangent plane to the surface $P_{R}(\mathbf{r})$, from which we extract the local gradient $[48,50]$. In the dynamical mode, which permits a faster evaluation of the measurement angle, we dynamically modulate the nanowire rest position in the $X Z$ planes at two different frequencies $(80$ and $85 \mathrm{~Hz})$ and demodulate the reflected signal. The amplitude and phase of both signals are then used to determine the local slope and orientations of the gradient along the two axes. Figure 12 shows a map of the measurement vectors measured during the multifrequency $X Z$ map shown in Fig. 4. The oscillation amplitudes employed for the dynamical calibration of the measurement vectors are of a few nanometers. These calibration tones are interrupted during the measurement phase, in order not to additionally shake the nanowire during the measurement process.

The fluctuations of the photodiode output are recorded on the spectrum and network analyzers, as shown in Fig. 8 and in Fig. 3 of the main text. In the absence of an external drive, we can record the thermal noise of the nanowire, which allows us to determine the effective mass $M_{\text {eff }}$, eigenfrequencies $\Omega_{1}, \Omega_{2}$, damping rate $\Gamma_{m}$, and eigenmode orientations $\mathbf{e}_{1}, \mathbf{e}_{2}$ of the two fundamental transverse eigenmodes [50]. In those experiments, we only rely on a single measurement channel, meaning that we have to assume that the eigenmode orthogonality is preserved [50,52]. In practice, we intentionally employ a nanowire presenting a large-frequency splitting $(50-60 \mathrm{kHz})$ to reduce the eigenmode rotation caused by the force field. After mapping the force field, we can verify that its spatial variations do not generate appreciable eigenmode rotations.

\section{Nanowire dynamics, thermal noise, and response measurements}

The nanowire dynamics can be written in the Fourier domain:

$$
\delta \mathbf{r}[\Omega]=\chi[\Omega] \cdot\left(\delta \mathbf{F}_{\text {th }}+\mathbf{F}_{\text {opt }}[\Omega]\right),
$$

where the $2 \mathrm{D}$ mechanical susceptibility is given by

$$
\chi^{-1}[\Omega] \equiv M_{\mathrm{eff}}\left(\begin{array}{cc}
\Omega_{1}^{2}-\Omega^{2}-i \Omega \Gamma_{\mathrm{m}} & 0 \\
0 & \Omega_{2}^{2}-\Omega^{2}-i \Omega \Gamma_{\mathrm{m}}
\end{array}\right)
$$


in the $\left(\mathbf{e}_{\mathbf{1}}, \mathbf{e}_{\mathbf{2}}\right)$ basis. The Langevin force vector $\delta \mathbf{F}_{\text {th }}=$ $\left(\delta F_{1}^{\text {th }}, \delta F_{2}^{\text {th }}\right)$ is composed of two Langevin forces, independently driving the nanowire along the eigenmode orientations. They present a zero mean value and a white-noise spectral density given by the fluctuation dissipation theorem of $S_{F} \equiv 2 M_{\mathrm{eff}} \Gamma_{\mathrm{m}} k_{B} T$. The optical force vector is given by $F_{\text {opt }}(t)$ and depends on the injected IR light intensity $P(t)$. It can be time modulated at a frequency $\Omega / 2 \pi$ with an amplitude $\delta P$ around a mean value $P_{0}$ using an acousto-optic modulator $P(t)=P_{0}+\delta P \cos (\Omega t)$. In that case, the optical force will present a static component (which can displace the nanowire rest position, but can be neglected in practice) and an oscillating counterpart $\delta \mathbf{F}_{\text {opt }} \cos (\boldsymbol{\Omega t}+\varphi)$. In the case of a pure optical force and in the adiabatic cavity limit $\left(\Omega_{\mathrm{m}} \ll \kappa\right)$ where we operate, the intracavity force instantaneously follows, on mechanical timescales, the time fluctuations of the input intensity $(\varphi=0)$. In the case of thermal-optical forces, the establishment of the force may be delayed. Previous analyses [48] have shown that the thermal dephasing is of 90 degrees at room temperature (thermal cutoff around $1 \mathrm{kHz}$ for the nanowires employed in that work). As such, the Fourier component of the time-modulated optical force vector can generally be written as

$$
\delta \mathbf{F}_{\text {opt }}[\Omega]=\delta F \mathbf{e}_{\mathbf{F}}+i \delta \tilde{F} \mathbf{e}_{\tilde{\mathbf{F}}} .
$$
by

The projective readout permits us to record $\delta r_{\beta}[\Omega]$ given

$\delta r_{\beta}[\Omega]=\mathbf{e}_{\beta} \cdot \delta \mathbf{r}[\Omega]=\mathbf{e}_{\beta} \cdot \chi[\Omega] \cdot\left(\delta \mathbf{F}_{\text {th }}+\mathbf{F}_{\text {opt }}[\Omega]\right)$.

In the absence of optical modulation, one can record the thermal noise of the nanowire, whose spectral density is given by

$S_{\delta r_{\beta}}[\Omega]=\sum_{i=1,2}\left(\mathbf{e}_{\beta} \cdot \mathbf{e}_{\mathbf{i}}\right)^{2} \frac{S_{F}}{M_{\mathrm{eff}}^{2}\left(\left(\Omega_{i}^{2}-\Omega^{2}\right)^{2}+\Omega^{2} \Gamma_{\mathrm{m}}^{2}\right)}$.

This expression is used to adjust the thermal noise spectra, and it allows us to determine the mechanical parameters of the nanowire (frequencies, damping rates, effective mass) and the effective temperature of the modes. One can verify their proper thermalization, at $300 \mathrm{~K}$, by varying the optical readout power. In the presence of the IR cavity light field, the mechanical properties can evolve because of the spatial gradients of the optical forces. If one uses a single measurement channel, one needs to assume that the eigenmodes remain orthogonal to correctly evaluate their response to an external force; this is why we employ a largely frequency split nanowire, which is protected against eigenmode rotations [52]. In practice, we realize optical measurements mainly on the reflected red channel, but we complement it using the scatter IR light and the IR transmission channel.
In the presence of an external optical drive, we employ a network analyzer to measure the steady-state trajectory of the nanowire while sweeping the drive frequency across both eigenmodes. To correctly estimate the overall delays in the VNA measurement, one can simply remove the IR filter in front of the detector collecting the probe-laser reflection (the WDM does not perfectly cancel the IR signal). This approach allows us to compensate for the electronic delays in the detection channel and in the drive channel. In that situation, we then directly record the magnitude of $\delta r_{\beta}[\Omega]$ and its dephasing with respect to the intensity modulation. The response data are thus adjusted by

$$
\delta r_{\beta}[\Omega]=\sum_{i=1,2} \frac{\left(\mathbf{e}_{\beta} \cdot \mathbf{e}_{\mathbf{i}}\right)\left(\delta \mathbf{F}[\Omega] \cdot \mathbf{e}_{\mathbf{i}}\right)}{M_{\mathrm{eff}}\left(\Omega_{i}^{2}-\Omega^{2}-i \Omega \Gamma_{\mathrm{m}}\right)} .
$$

All of the mechanical and readout parameters are already independently determined, so the only fitting parameter is the complex drive vector $\delta \mathbf{F}[\Omega]$, which possesses 4 degrees of freedom (real and imaginary parts of both spatial components). In practice, we employ expression (B3), so the fitting parameters are the magnitudes $(\delta F, \delta \tilde{F})$ and orientations $\left(\mathbf{e}_{\mathbf{F}}, \mathbf{e}_{\tilde{\mathbf{F}}}\right)$ of the instantaneous and delayed force components. They are shown in Fig. 9. The gradients of the delayed photothermal forces modify the nanowire damping matrix, so the nanowire mechanical damping rates must be determined in each position. At the end of the article, we focus on the instantaneous optical force, the one of interest in cavity optomechanics, whose spatial gradients can generate mechanical frequency shifts and eigenmode rotations, as analyzed in Fig. 3(f).
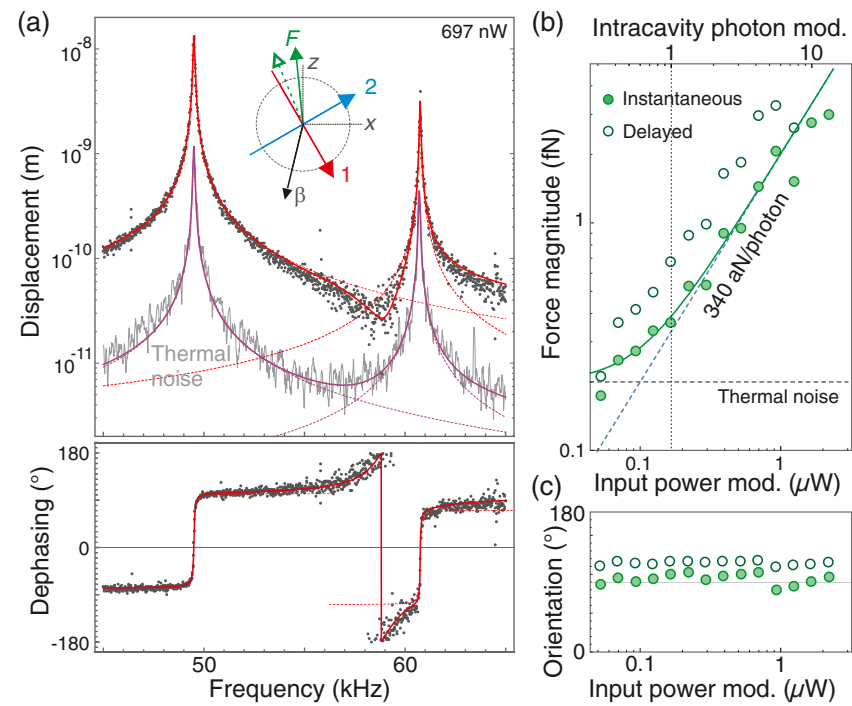

FIG. 9. Delayed photothermal forces. We add the delayed optical forces, of photothermal origin, to Fig. 3. The open symbols represent the magnitudes and orientations of the delayed force vectors, at the measurement position, for increasing modulation depth. The solid symbols represent the instantaneous optical forces. 


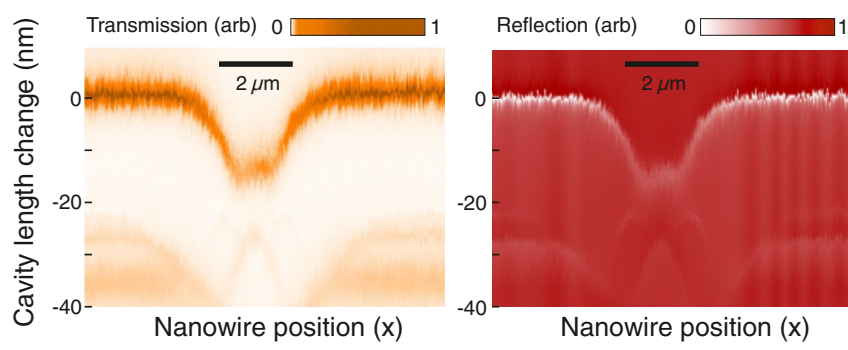

FIG. 10. Nanowire-based scanning probe exploration of the intracavity field and higher-order transverse modes. Cavity IR transmission and reflection stacks are measured while scanning its total length with the slow piezo and moving the nanowire across the optical mode ( $x$ axis). For this cavity configuration, in addition to the $\mathrm{TEM}_{00}$ mode (top), higher-order modes are also visible (bottom), and cross-couplings can be induced by the nanowire diffusion.

\section{Higher-order optical cavity modes}

It is possible to investigate the optomechanical coupling between the nanowire and higher-order transverse modes of the cavity, whose transverse structure can be identified by scanning the nanowire within the cavity mode (see Fig. 10). The optomechanical force field experienced when pumping the $\mathrm{TEM}_{12}$ mode is shown in Fig. 11.

\section{Multifrequency response measurements}

The above procedure based on full response measurements requires at least a duration of $1 / \Gamma_{\mathrm{m}}$ per frequency point $(100 \mathrm{~ms})$, so the full acquisition requires at least 20 30 s per point before fitting the response data, which allows us to determine the local optical force vector. It is possible to largely accelerate the measurement duration by exploiting the large linearity of the apparatus and driving the nanowire simultaneously with several (6) frequencies and demodulating the different driven trajectories. In order to simultaneously determine the eigenmode frequencies and damping rates and to compensate for possible variations due to force-field gradients or thermal drifts, we choose to employ three tones per mechanical mode, at frequencies $\Omega_{i}^{0, \pm 1}$ separated by $50 \mathrm{~Hz}$, which is comparable to the eigenmode linewidth (see Fig. 13). The central frequency $\Omega_{i}^{0}$ is maintained close to the mechanical resonance using a soft tracking via a peak detection mode on a fast spectrum analyzer. These measurements are realized using a multifrequency synchronous detector (Zurich Instrument HF2LI), and they permit us to reduce the measurement duration to $100 \mathrm{~ms}$ per point, at least 100 times faster than the above response measurements.

The interference pattern measured in the reflection of the red probe laser, which is exploited to read out the nanowire vibrations, presents positions (interference extrema) featuring no spatial gradient, which prevent us from correctly measuring the nanowire vibrations. It is important to properly estimate the local measurement vectors, but this

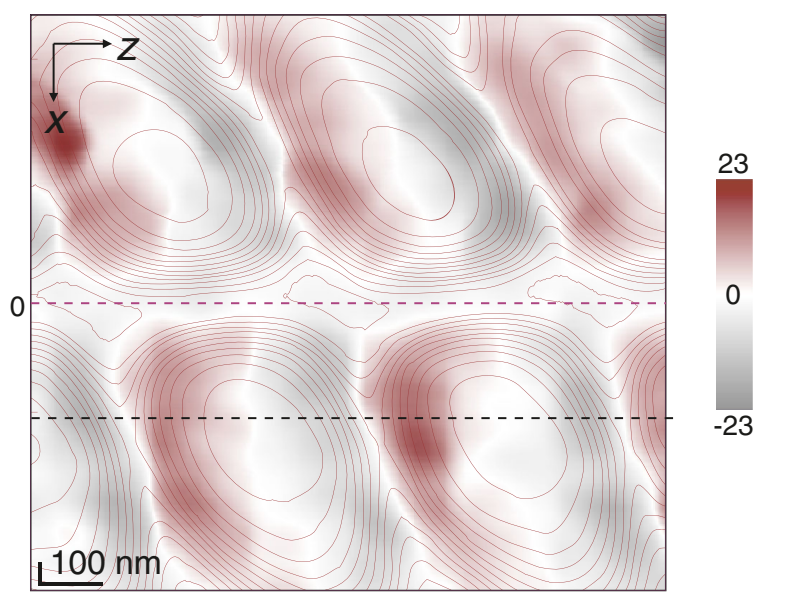

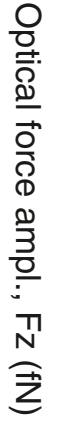
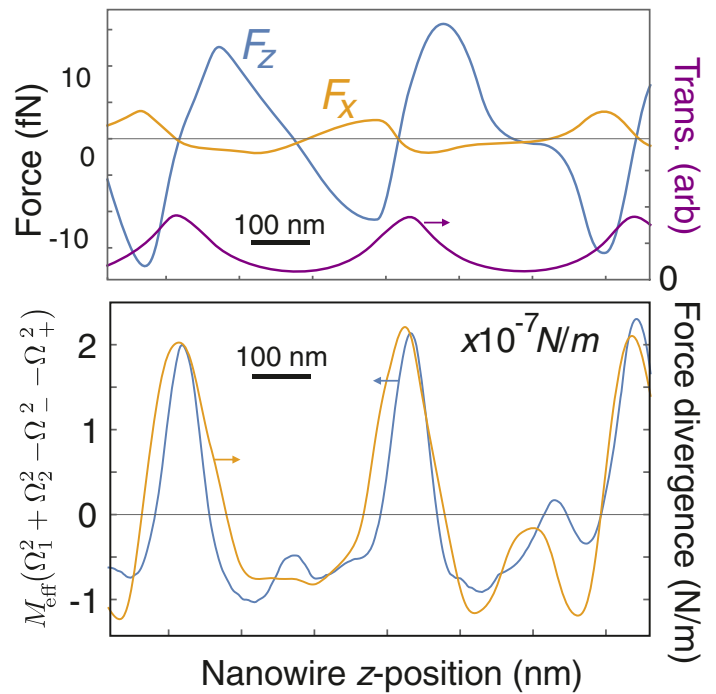

FIG. 11. Optomechanical force field obtained with a TEM 12 mode. Top: multifrequency force-field measurement realized when locking the cavity on a higher-order transverse mode. The map is interpolated from a $400 \times 400$ measurement grid. The gray lines are isotransmission lines. Middle: cuts along the $z$ direction (dashed line in the map, on the side of the optical axis) of the force components. Bottom: force divergence deduced from mechanical frequency shifts (blue) and spatial derivation of the force map (orange), evaluated along the same cut in the map.

may become delicate close to those extrema. In order to compensate for this potential source of errors, we have applied a local barycentric correction to the data: The force measurements are spatially averaged and weighted by the norm of the measurement vector magnitude:

$$
F_{i}^{\mathrm{avg}}=\frac{\sum_{j=i-\Delta, i+\Delta} F_{j}\left|\beta_{j}\right|}{\sum_{j=i-\Delta, i+\Delta}\left|\beta_{j}\right|}
$$

where $F_{i}$ represents the force estimated at position $i$. We employ a sliding average over $2 \Delta+1$ neighboring points spreading over $25 \mathrm{~nm}$ ( $\Delta$ is adjusted depending on the map grid). Figure 14 illustrates this mechanism. The upper curve represents the cavity transmission, which allows us to 

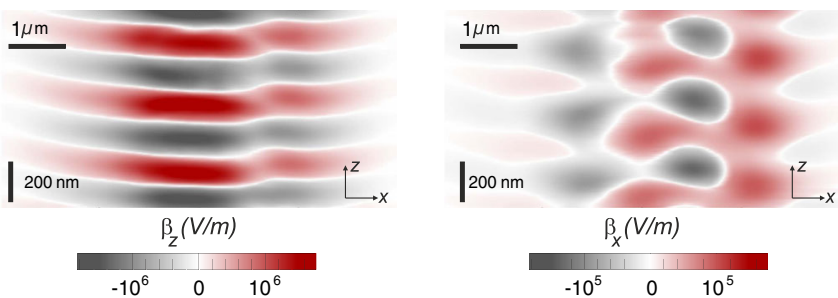

FIG. 12. Maps of the measurement vectors. We shows the $X Z$ maps of the measurement vectors $\left(\beta_{z}, \beta_{x}\right)$ obtained with the spatial interference measured on the red-probe-laser reflection photodiode signal $P_{R}\left(\mathbf{r}_{\mathbf{0}}\right)$. Measurement vectors defined as $\boldsymbol{\beta} \equiv$ $\nabla P_{R}$ are measured dynamically, by modulating the piezo stage supporting the nanowire with constant tones at 80 and $85 \mathrm{~Hz}$ along the $x$ and $z$ axes (approximately $5 \mathrm{~nm}$ of amplitude) and demodulating the photodiode output signal $(1-10 \mu \mathrm{W}$ of $633-\mathrm{nm}$ probe laser are typically employed). Those measurements are acquired simultaneously during the multifrequency response maps shown in Fig. 4 of the main text, on a $400 \times 400$ pixel map, with $100 \mathrm{~ms}$ acquisition time per point. The $z$ periodicity observed corresponds to half the probe-laser wavelength $(633 \mathrm{~nm})$. In most positions, the measurement vectors $\mathbf{e}_{\beta}=$ $\boldsymbol{\beta} /|\boldsymbol{\beta}|$ are almost aligned with the optical axis $\mathbf{e}_{\mathbf{z}}$.

identify nodes and antinodes of the intracavity field; the bottom curve represents the norm of the measurement vector, which presents blind positions (marked by gray dashed lines). The central panel shows the force measured before (dots) and after (line) the barycentric correction. We note that because of the large signal-to-noise ratio observed in our measurement conditions (force signal $20 \mathrm{~dB}$ above the thermal noise and $60 \mathrm{~dB}$ above the background for $5-\mathrm{Hz}$ resolution bandwidth), it is always possible to detect the driven force, even very close to the blind positions. The barycentric averaging thus serves to compensate for the errors arising in the evaluation of the measurement vectors close to the blind positions.

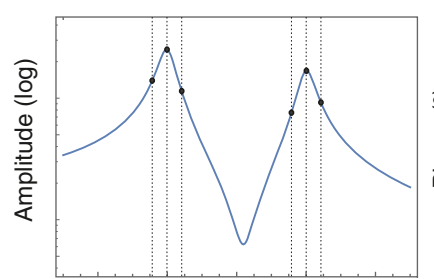

Frequency

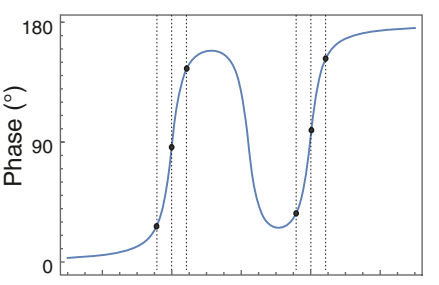

Frequency

FIG. 13. Principle of multifrequency response. The nanowire is simultaneously driven by the intracavity field at six different frequencies, in order to accelerate the measurement time of the local force vector. Three tones are driving each eigenmode, the central tone of which is locked on the eigenmode resonance, while the two sidebands are separated by $50 \mathrm{~Hz}$, which is comparable to the eigenmode linewidth. Recording the amplitudes and dephasing for each tone permits us to determine the quality factors and eigenmode frequencies, as well as the local complex driving force vector.

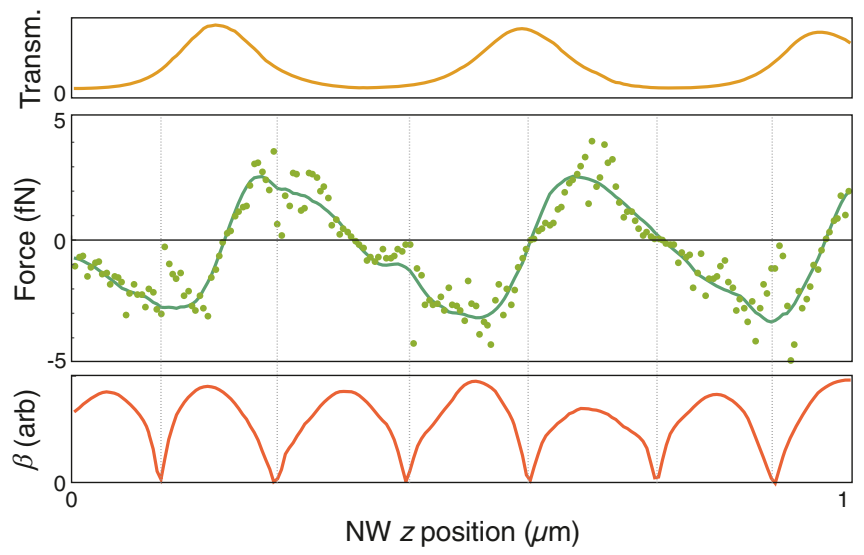

FIG. 14. Correction for blind measurement positions in multifrequency responses. We give an illustration of the local barycentric smoothing employed in the data analysis to compensate for the reduction of readout sensitivity observed in the readout channel at the interference extrema. The cavity transmission is shown on top, while the bottom curves represent the norm of the measurement vector. Blind positions are marked by gray dashed lines. The central panel shows the force measured before (dots) and after (line) the local weighted averaging explained in the text.

\section{APPENDIX C: MODELING}

\section{Orders of magnitude: Coarse estimation}

The change in path length $\Delta l$ accumulated in the presence of the nanowire can be roughly evaluated as a geometric evaluation, where part of the vacuum is replaced by the nanowire dielectric. When the nanowire of diameter $d_{\text {nw }}$ is positioned in the middle of a Gaussian beam of waist $w_{0}$, it can be expressed as

$$
\Delta l=\left(n_{S i C}-1\right) d_{\mathrm{nw}} \frac{2}{\pi w_{0}^{2}} \int_{-d_{\mathrm{nw}} / 2}^{d_{\mathrm{nw}} / 2} d x \int_{-\infty}^{\infty} d y e^{-2\left(x^{2}+y^{2}\right) / w_{0}^{2}} .
$$

Thus, the relative cavity path-length change amounts to $\Delta l / L=1.1 \times 10^{-3} \quad$ for $\quad d_{\mathrm{nw}}=130 \mathrm{~nm}, \quad w_{0}=1.7 \mu \mathrm{m}$, $n_{\mathrm{SiC}}=2.7$, and $L=12 \mu \mathrm{m}$. This order of magnitude is in good agreement with our measurements (see main text).

\section{Model}

Here, we briefly describe the ingredients employed in the model. It is based on a transmission matrix formulation, as standardly employed for membrane-in-the-middle experiments $[37,62]$, with the difference that one has to describe a nanowire instead of a membrane.

The waist and the Rayleigh length of the cavity mode are determined by the cavity length, the mirror curvature radii, and the laser wavelength. For simplicity, the cavity is assumed to be only resonant with the $\mathrm{TEM}_{00}$ Gaussian mode. In practice, cross-couplings between the different eigenmodes are easily obtained and can be employed for enhancing the quadratic coupling strength if needed, but the experimental results exposed in this paper were obtained in 
a situation where no cross-couplings were observed. The cavity is pumped from the left side. Several approaches were employed to compute the transfer matrix of the nanowire, with different degrees of refinement (possibility of transverse coupling, description of the internal Mie resonances of the nanowire,...). A simple approach is described below, which permits us to understand most of the experimental signatures we have observed.

We assume that the nanowire dimensions are sufficiently small compared to the optical waist, so the incoming field presents wavefronts that are homogeneous over the nanowire extension and thus employ a "square" nanowire of lateral dimensions $L_{\mathrm{NW}} \times L_{\mathrm{NW}}$. To integrate the nanowire in the input-output formalism, we need to compute its effective reflection, transmission, and loss coefficients. We thus define a nanowire effective layer, with reflection and transmission coefficients, that depends on $\mathbf{r}_{\perp}$, the transverse coordinate. When $\mathbf{r}_{\perp}$ belongs to the nanowire, these coefficients are taken to be identical to the reflection and transmission coefficients of the equivalent infinite layer, with refractive index $n$ and thickness $L_{\mathrm{NW}}$ :

$$
\begin{aligned}
C_{r} & =\frac{\left(1-n^{2}\right) \sin \left(n k L_{\mathrm{NW}}\right)}{2 i n \cos \left(n k L_{\mathrm{NW}}\right)+\left(1+n^{2}\right) \sin \left(n k L_{\mathrm{NW}}\right)}, \\
C_{t} & =\frac{2 i n}{2 i n \cos \left(n k L_{\mathrm{NW}}\right)+\left(1+n^{2}\right) \sin \left(n k L_{\mathrm{NW}}\right)} .
\end{aligned}
$$

If $\mathbf{r}_{\perp}$ does not belong to the nanowire, we assume a planewave propagation (which is valid for a nanowire close to the middle of the cavity) such as $C_{r}=0$ and $C_{t}=\mathrm{e}^{i k L_{\mathrm{NW}}}$. The final reflection and transmission coefficients of the Gaussian beam through the nanowire effective layer are obtained by assuming that the only parts of the fields contributing to the intracavity field are the projections on the $\mathrm{TEM}_{00}$ mode since they are the only ones assumed to be resonant inside the cavity. They read as

$$
\begin{aligned}
& t_{00}^{00}=\iint \mathrm{d} \mathbf{r}_{\perp} E_{t}\left(\mathbf{r}_{\perp}, Z_{\mathrm{NW}}\right)\left[E_{(+)}^{00}\left(\mathbf{r}_{\perp}, Z_{\mathrm{NW}}\right)\right]^{*}, \\
& r_{00}^{00}=\iint \mathrm{d} \mathbf{r}_{\perp} E_{r}\left(\mathbf{r}_{\perp}, Z_{\mathrm{NW}}\right)\left[E_{(-)}^{00}\left(\mathbf{r}_{\perp}, Z_{\mathrm{NW}}\right)\right]^{*},
\end{aligned}
$$

where $E_{( \pm)}^{00}$ are the $\mathrm{TEM}_{00}$ normalized modes $\left[\iint \mathrm{d} \mathbf{r}_{\perp}\left|E_{( \pm)}^{00}\left(\mathbf{r}_{\perp}, z\right)\right|^{2}=1\right]$ propagating along $\pm z$.

In practice, the simulations are done in two steps. First, we compute the transfer matrix of the nanowire effective layer for all positions of the nanowire inside the cavity (both axially and vertically). Then, we use these transfer matrices to propagate the field through the system, obtaining the transmission and reflection coefficients of the full cavity (see Figs. 15 and 16). Using the energy conservation, we also extract the amount of light scattered out of the cavity due to the presence of the nanowire.

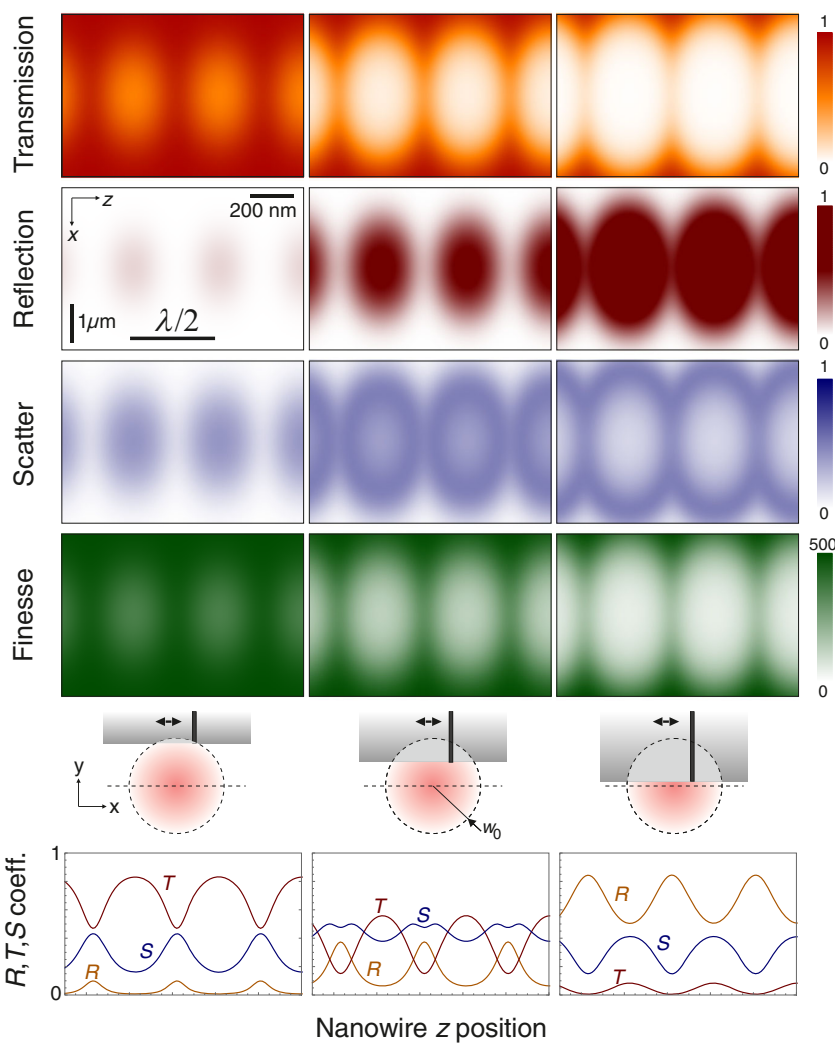

FIG. 15. Typical results of simulations. The transmitted, reflected, and scattering coefficients, as well as the cavity finesse, are computed for various positions of the nanowire in the $X Z$ plane and for three different vertical insertions in the mode volume. From left to right: the nanowire bottom extremity is located at 1.8, 1, and $0.2 \mu \mathrm{m}$ above the optical axis (optical waist of $\left.w_{0}=1.8 \mu \mathrm{m}\right)$. The other bare cavity properties are 500 finesse, symmetric mirrors, and $12 \mu \mathrm{m}$ length.

Those simulations are realized for different laser frequencies (or, equivalently, for different cavity lengths) in the vicinity of the system resonance, which can be used to compute the strength of the parametric shifts of the cavity as well as modifications of its linewidth. Also, it is possible to track the cavity resonance to compute the optical signal or optical forces in the presence of a cavity lock, as realized experimentally [Figs. 2(d), 2(e), 3, and 4].

Moreover, we subsequently determine the field inside the two subcavities surrounding the nanowire (see Fig. 17). Once the fields around the nanowire are known, we can estimate the optical force exerted by the intracavity field on the nanowire and, in particular, its impact on the dynamics of the two transverse fundamental eigenmodes of the nanowire. To do so, one needs to convolve the optical force density with the eigenmode deformation profile. Since the optical field is essentially located close to its vibrating extremity, where the eigenmode deformation profiles are locally uniform and can be assimilated to a simple translation on the extent of the optical waist, the effect of the optical forces can be assimilated to the integral 


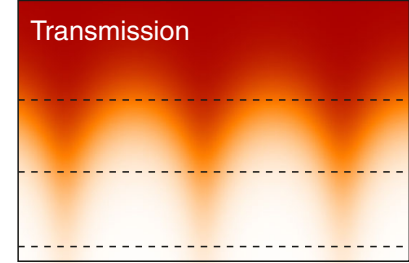

0

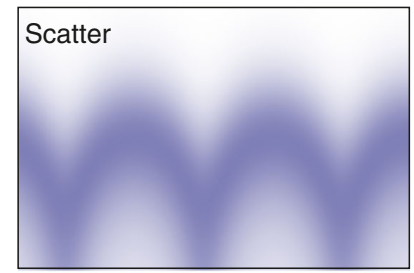

0

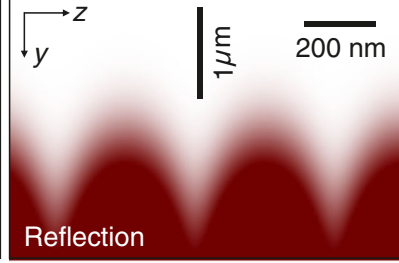

0

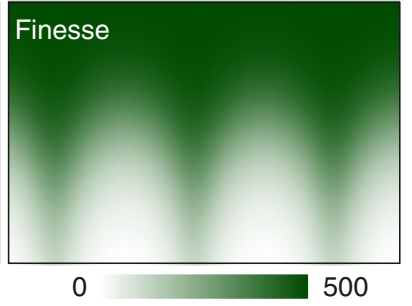

FIG. 16. Typical results of simulations. Same simulations as in Fig. 15 in the vertical $Y Z$ plane above the optical axis $(x=0)$. The optical axis is located at the bottom of the image, and the three dashed lines correspond to the NW elevations in the previous figure.

of the Maxwell stress tensor on the nanowire surface. Assuming a nanowire positioned on the cavity axis, we can then compute the optical force along $z$ applied by the intracavity field on the nanowire for different positions and cavity properties.

Figure 17 shows a typical result of the axial force-field pattern measured for varying positions along the optical axis obtained with this modeling (square nanowire of 115-nm dimensions). The right panels illustrate the spatial profiles of the electromagnetic field intensity in the system, for two positions of the oscillator on the left and on the right of a node. For both positions, the parametric shift is identical, but the optical force changes sign. This case can be explained by the different amount of light stored in the left or right subcavities. Furthermore, the positive or negative asymmetry observed in the force profile is due to the asymmetric pumping conditions: It is easier to pump the left cavity
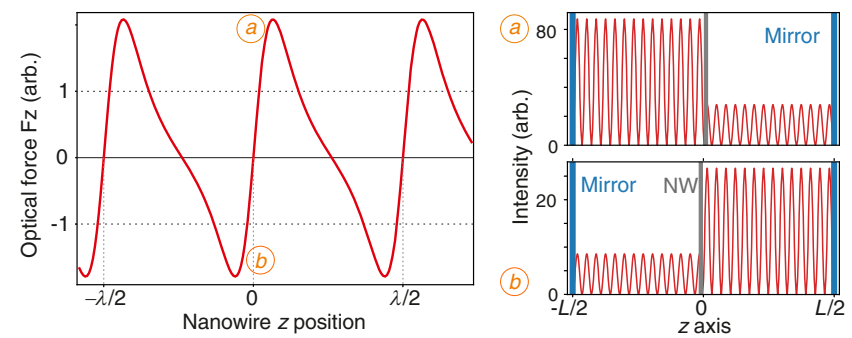

FIG. 17. Simulations of the optical axial force computed for different nanowire positions along the optical axis. The right panels illustrate the shape of the intensity distribution in the system for two positions of the oscillator, on the left and right sides of an optical node of the intracavity field. The left-right distributions of the field intensity in the two subcavities allow us to understand the repulsive character of the force profile observed in the vicinity of optical nodes. compared to the right cavity when the system is pumped from the left-hand side. In those simulations, both mirrors were taken to be identical; in practice, this asymmetric behavior also depends on the mirror reflectivities.

The simulated and experimentally measured force fields are observed to be very repulsive in the vicinity of the optical nodes (large slopes) but, respectively, less attractive to the optical antinodes (smaller inverse slopes) even if the parametric coupling strength $G_{z}$ presents the same magnitude at both positions. This is a consequence of the reduction of the cavity finesse observed when the nanowire enters the scatter rings visible in the scatter maps: Therefore, the intracavity field benefits from a lower cavity enhancement, thus reducing the magnitude of the optical force exerted on the nanowire.

\section{APPENDIX D: INTRACAVITY PHOTON NUMBER}

The intracavity photon numbers employed in the response measurements are experimentally deduced using the input-output formalism, modeling the overall nanowirein-the-middle system as an equivalent, dual-port, 1D optomechanical system with an input mirror of transmissivity $T$ (in intensity) and a back mirror featuring a transmissivity $P$ (taking into account the second mirror transmissivity as well as the nanowire-induced optical losses). The intracavity photon number $N$ is related to the intracavity photon flux $I$ (in photon/s) by $I=N /(2 L / c)$, where $2 L / c$ is the photon round-trip duration in the cavity, while the latter is related to the input photon flux $I_{\text {in }}=P_{\text {in }} / h \nu$ by $I=I_{\text {in }}\left[4 T /(T+P)^{2}\right]$ in the case of a perfect injection efficiency. The cavity finesse is connected to the overall cavity losses by $\mathcal{F}=2 \pi /(T+P)$. In our experiment, the injection efficiency $\eta$ is limited by the fiber injection efficiency $(80 \%$ including splices and fiber connector losses) and the mode matching from the fiber to the cavity fundamental mode (70\%) to an overall value of $\eta=0.56$. The connection between the incoming power $P_{\text {in }}$ and the intracavity photon number is thus given by

$$
N=\eta \frac{2 L}{\pi c} \mathcal{F} \frac{2 T}{T+P} \frac{P_{\text {in }}}{h \nu}=\eta \frac{2 L}{\pi^{2} c} \mathcal{F}^{2} T \frac{P_{\text {in }}}{h \nu} .
$$

The cavity parameters $(T, P)$ are deduced from optical measurements of the cavity finesse realized by scanning the cavity length around a resonance. In the absence of the nanowire, we operate with a symmetric cavity $(P=T)$, while the cavity external losses $P$ are increased in the presence of the nanowire and are computed in each position. In Figs. 3(a)-3(d), at the position where the response linearity is verified, a modulation of the input power of $100 \mathrm{nW}$ generates a modulation of the intracavity photon number of $0.6[\mathcal{F}=202,4 T /(T+P)=1.05, L=12 \mu \mathrm{m}]$, so we can largely detect modulations of the mean intracavity photon number smaller than unity. 
The advanced numerical simulations [58] of the system (see Fig. 18) show that this assimilation of the nanowire-inthe-middle setup to an equivalent 1D optomechanical system is valid to estimate the intracavity photon number in the case of a response measurement where the system is pumped from one single port. In this approach, after having evaluated the intracavity fields, the photon number is deduced by computing the overall energy stored in the optical resonator and dividing it by the energy of a single photon $(h \nu)$. The numerical simulations also permit us to estimate the expected force per intracavity photon change, at the level of $260 \mathrm{aN} /$ photon, whose magnitude is in good agreement with our measurements (340 aN/photon). We underline the fact that the model employed here assumes that the nanowire optical response can be assimilated to the one of an infinite cylinder (so the description of light scattering in the framework of Mie scattering remains analytical), inserted perfectly transversally to the optical mode. In practice, we operate, in general, only a few microns above the nanowire tip, which may not be sufficiently far away from the tip, and we often suffer from a residual tilt (typically a few degrees) of the nanowire with respect to the optical axis. Finally, the delicate estimation of the optical pumping efficiency will always remain one of the most important sources of discrepancy between our measurements and the ideal predictions of the model.
In both the theoretical and experimental cases, those single-photon forces are comparable to but about 0.4 times smaller than the value of $F^{(1)}$ expected from the coupling strength $g_{0}$. The understanding of the origin of this slight discrepancy and the modeling of the contribution of all the other vacuum modes (Casimir forces) will be the subject of future work.

\section{APPENDIX E: OBSERVING QUANTUM RADIATION-PRESSURE NOISE AT THE SINGLE-PHOTON LEVEL}

The goal of this section is to derive, in the semiclassical framework, a dynamical criterion comparing the impact of the radiation-pressure force fluctuations $\delta F_{\mathrm{i}}$ and of the thermal fluctuations $\delta F_{\text {th }}$ on the oscillator dynamics in an optomechanical system. For that purpose, we consider the simple case of a high-finesse and singleport optical cavity pumped by a monochromatic laser, where the second mirror can oscillate around its equilibrium position. Following the methodology of Ref. [5], the oscillator displacement in frequency space is shown to be

$$
\delta x[\Omega]=\chi_{\mathrm{eff}}[\Omega]\left(\delta F_{\mathrm{th}}[\Omega]+\delta F_{\mathrm{rad}}[\Omega]\right)
$$
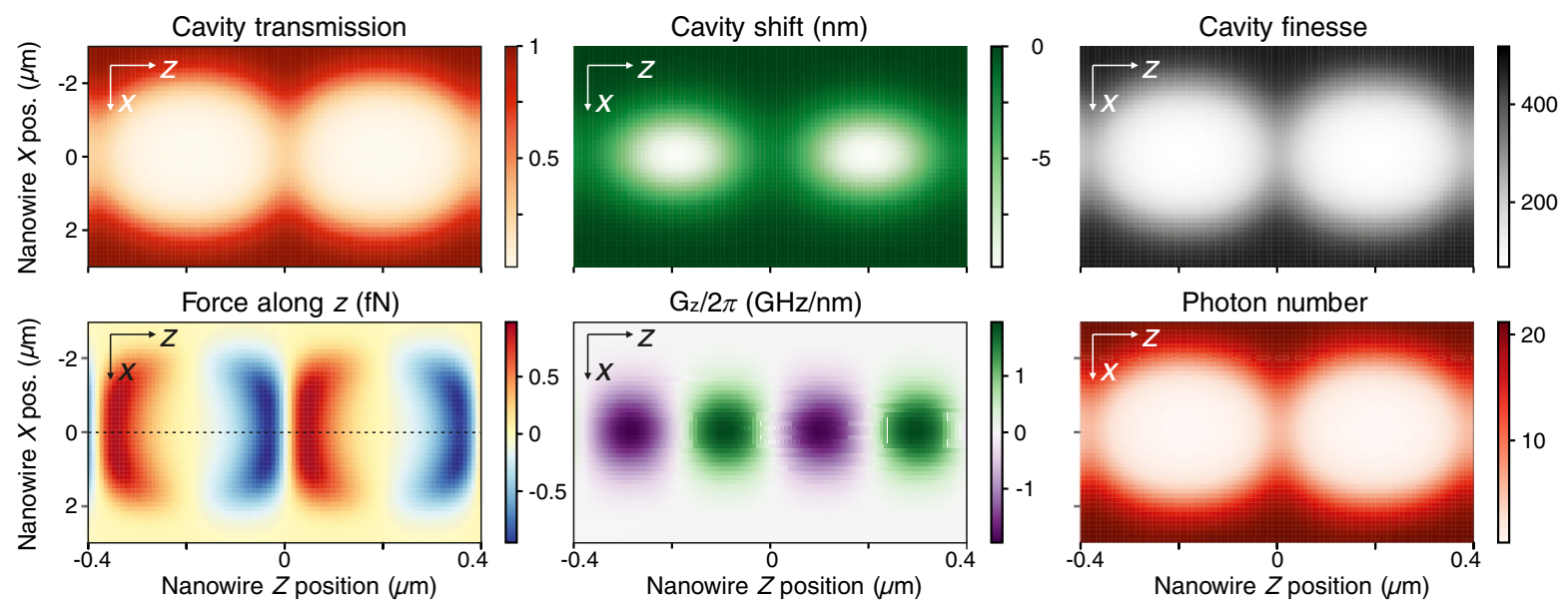

Photon number on the cavity axis
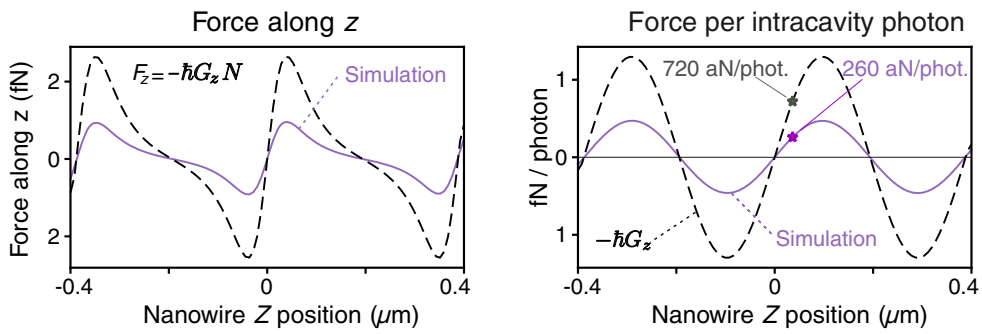

FIG. 18. Numerical simulations of the experiment. We show the results of the complete simulation [58] of the experiment, accounting for internal Mie resonances in the nanowire. We employ the following parameters: $L_{\mathrm{cav}}=12 \mu \mathrm{m} ; R_{\mathrm{nw}}=65 \mathrm{~nm}$; radius of curvature of the mirrors, $28 \mu \mathrm{m}$; mirrors reflectivities, 0.994; perpendicular polarization; wavelength $\lambda=770 \mathrm{~nm}$; and input power $P_{\text {inc }}=400 \mathrm{nW}$, with perfect mode matching. The lower panels are slices taken along the optical axis. 
where $\chi_{\text {eff }}$ is the effective susceptibility of the oscillator dressed by the light verifying $\chi_{\mathrm{eff}}[-\Omega]=\chi_{\mathrm{eff}}^{*}[\Omega]$. The expression of the radiation-pressure force fluctuations is

$$
\begin{aligned}
\delta F_{\text {rad }}[\Omega]= & 2 \hbar k|\bar{\alpha}| \sqrt{\frac{2 \gamma}{\gamma^{2}+\bar{\Psi}^{2}}}\left(\frac{\gamma^{2}+\bar{\Psi}^{2}-i \gamma \Omega \tau}{(\gamma-i \Omega \tau)^{2}+\bar{\Psi}^{2}} \delta p_{\text {in }}[\Omega]-i\right. \\
& \left.\times \frac{\bar{\Psi} \Omega \tau}{(\gamma-i \Omega \tau)^{2}+\bar{\Psi}^{2}} \delta q_{\text {in }}[\Omega]\right)
\end{aligned}
$$

where $\delta p_{\text {in }}$ and $\delta q_{\text {in }}$ are the quadratures of the intensity and phase fluctuations, whose noise spectral densitites will be set to 1 since, in the following, we consider a cavity pumped with a coherent state. In Eq. (E2), $k$ is the wave vector of light, $|\bar{\alpha}|^{2}$ the intracavity photon flux, $\gamma \ll$ 1 characterizes the cavity losses $(r=1-\gamma, t \simeq \sqrt{2 \gamma}), \tau=$ $2 L / c$ is the intracavity round-trip time, and $\bar{\Psi}$ is the cavity-laser detuning.

Since $\delta F_{\text {rad }}$ and $\delta F_{\text {th }}$ are uncorrelated, the position noise spectrum reads as

$$
S_{\delta_{x}}[\Omega]=\left|\chi_{\mathrm{eff}}[\Omega]\right|^{2}\left(S_{\delta F_{\mathrm{rad}}}[\Omega]+S_{\delta F_{\mathrm{rad}}}[\Omega]\right),
$$

so the quantum radiation-pressure noise will dominate if the following criterion is fulfilled:

$$
\eta_{\mathrm{rad}} \equiv S_{\delta F_{\text {rad }}}[\Omega] / S_{\delta F_{\mathrm{th}}}[\Omega]>1 .
$$

In the case of an adiabatic cavity $\left(\Omega_{m} \ll \Omega_{c}\right.$, with $\Omega_{c}$ being the cavity cutoff frequency), the noise spectral density of the optical force is given by

$$
S_{\delta F_{\mathrm{rad}}}[\Omega]=\frac{8 \hbar^{2} k^{2}|\bar{\alpha}|^{2} \gamma}{\gamma^{2}+\bar{\Psi}^{2}},
$$

while the Langevin noise spectral density is $S_{\delta F}[\Omega]=2 M \Gamma_{\mathrm{m}} k_{B} T$, where $\mathrm{M}$ is the effective mass of the oscillator, $\Gamma_{\mathrm{m}}$ its decoherence rate, and $T$ the temperature of the bath. At the optical resonance $(\bar{\Psi}=0)$, the dynamic criterion becomes

$$
\eta_{\mathrm{rad}}=\frac{4 \hbar^{2} k^{2}|\bar{\alpha}|^{2}}{M \Gamma_{\mathrm{m}} k_{B} T \gamma}
$$

where $|\bar{\alpha}|^{2}=N c / 2 L$ is expressed in terms of the intracavity photon number $N$ and of the cavity length $L$ ( $c$ being the light velocity). Using $\gamma / k=\kappa / G$ and $L / k=1 / G$, with $G=\omega / L=\partial_{x} \omega$ the optomechanical coupling strength of the system, we get

$$
\eta_{\mathrm{rad}}=2 N C^{(1)} \frac{Q}{n_{T}},
$$

where we have also used $g_{0}=G \delta x_{\mathrm{zpf}}$ and the expression of the single-photon optomechanical parametric cooperativity
$C^{(1)} \equiv 2 g_{0}^{2} / \kappa \Omega_{m}$ introduced in the main text. In Eq. (E7), $n_{T} \equiv k_{B} T / \hbar \Omega_{m}$ is the initial thermal phonon number, and $Q=\Omega_{m} / \Gamma_{\mathrm{m}}$ is the nanowire quality factor. In the case of an adiabatic cavity, the latter is only weakly affected by the optomechanical effects, and it can be assimilated to its value in the absence of light.

When the system is bistable at the single-photon level, $C^{(1)}>1$, the dynamical criterion simply requires $Q>n_{T} / 2$. Both conditions would be fulfilled if one could reproduce the present experiment in a dilution fridge, using the nanowires already employed in such a cold environment [60]: $2 Q / n_{\mathrm{T}}$ ratios above 2.5 were already achieved, while cooperativities larger than 10 can be envisioned. This experiment is challenging but feasible, and the orders of magnitude are clearly encouraging.

[1] M. Aspelmeyer, T. J. Kippenberg, and F. Marquardt, Cavity Optomechanics, Rev. Mod. Phys. 86, 1391 (2014).

[2] V. B. Braginsky, F. Y. Khalili, and K. S. Thorne, Quantum Measurement (Cambridge University Press, Cambridge, England, 1995).

[3] C. M. Caves, Quantum-Mechanical Radiation-Pressure Fluctuations in an Interferometer, Phys. Rev. Lett. 45, 75 (1980).

[4] M. T. Jaekel and S. Reynaud, Quantum Limits in Interferometric Measurements, Europhys. Lett. 13, 301 (1990).

[5] C. Fabre, M. Pinard, S. Bourzeix, A. Heidmann, E. Giacobino, and S. Reynaud, Quantum-Noise Reduction Using a Cavity with a Movable Mirror, Phys. Rev. A 49, 1337 (1994).

[6] J. Chan, T. P. M. Alegre, A. H. Safavi-Naeini, J. T. Hill, A. Krause, S. Gröblacher, M. Aspelmeyer, and O. Painter, Laser Cooling of a Nanomechanical Oscillator into Its Quantum Ground State, Nature (London) 478, 89 (2011).

[7] J. D. Teufel, T. Donner, D. Li, J. W. Harlow, M. S. Allman, K. Cicak, A. J. Sirois, J. D. Whittaker, K. W. Lehnert, and R. W. Simmonds, Sideband Cooling of Micromechanical Motion to the Quantum Ground State, Nature (London) 475 , 359 (2011).

[8] T. P. Purdy, R. W. Peterson, and C. A. Regal, Observation of Radiation Pressure Shot Noise on a Macroscopic Object, Science 339, 801 (2013).

[9] R. W. Peterson, T. P. Purdy, N. S. Kampel, R. W. Andrews, P. L. Yu, K. W. Lehnert, and C. A. Regal, Laser Cooling of a Micromechanical Membrane to the Quantum Backaction Limit, Phys. Rev. Lett. 116, 063601 (2016).

[10] N. S. Kampel, R. W. Peterson, R. Fischer, P. L. Yu, K. Cicak, R. W. Simmonds, K. W. Lehnert, and C. A. Regal, Improving Broadband Displacement Detection with Quantum Correlations, Phys. Rev. X 7, 021008 (2017).

[11] M. Rossi, D. Mason, J. Chen, Y. Tsaturyan, and A. Schliesser, Measurement-Based Quantum Control of Mechanical Motion, Nature (London) 563, 53 (2018).

[12] S. Weis, R. Rivière, S. Deléglise, E. Gavartin, O. Arcizet, A. Schliesser, and T. J. Kippenberg, Optomechanically Induced Transparency, Science 330, 1520 (2010). 
[13] E. Verhagen, S. Delglise, S. Weis, A. Schliesser, and T. Kippenberg, Quantum-Coherent Coupling of a Mechanical Oscillator to an Optical Cavity Mode, Nature (London) 482, 63 (2012).

[14] T. a. Palomaki, J. D. Teufel, R. W. Simmonds, and K. W. Lehnert, Entangling Mechanical Motion with Microwave Fields, Science 342, 710 (2013).

[15] F. Lecocq, J. B. Clark, R. W. Simmonds, J. Aumentado, and J. D. Teufel, Quantum Nondemolition Measurement of a Nonclassical State of a Massive Object, Phys. Rev. X 5, 041037 (2015).

[16] R. Riedinger, S. Hong, R. A. Norte, J. A. Slater, J. Shang, A. G. Krause, V. Anant, M. Aspelmeyer, and S. Gröblacher, Non-classical Correlations between Single Photons and Phonons from a Mechanical Oscillator, Nature (London) 530, 313 (2016).

[17] V. Sudhir, D. J. Wilson, R. Schilling, H. Schutz, S. A. Fedorov, A. H. Ghadimi, A. Nunnenkamp, and T. J. Kippenberg, Appearance and Disappearance of Quantum Correlations in Measurement-Based Feedback Control of a Mechanical Oscillator, Phys. Rev. X 7, 011001 (2017).

[18] J.-M. Pirkkalainen, E. Damskägg, M. Brandt, F. Massel, and M. A. Sillanpää, Squeezing of Quantum Noise of Motion in a Micromechanical Resonator, Phys. Rev. Lett. 115, 243601 (2015).

[19] G. A. Peterson, S. Kotler, F. Lecocq, K. Cicak, X. Y. Jin, R. W. Simmonds, J. Aumentado, and J. D. Teufel, Ultrastrong Parametric Coupling between a Superconducting Cavity and a Mechanical Resonator, Phys. Rev. Lett. 123, 247701 (2019).

[20] S. Reynaud, C. Fabre, E. Giacobino, and A. Heidmann, Photon Noise Reduction by Passive Optical Bistable Systems, Phys. Rev. A 40, 1440 (1989).

[21] S. Bose, K. Jacobs, and P. L. Knight, Preparation of Nonclassical States in Cavities with a Moving Mirror, Phys. Rev. A 56, 4175 (1997).

[22] S. Mancini, V. Man'ko, and P. Tombesi, Ponderomotive Control of Quantum Macroscopic Coherence, Phys. Rev. A 55, 3042 (1997).

[23] M. Ludwig, B. Kubala, and F. Marquardt, The Optomechanical Instability in the Quantum Regime, New J. Phys. 10, 095013 (2008).

[24] P. Rabl, Photon Blockade Effect in Optomechanical Systems, Phys. Rev. Lett. 107, 063601 (2011).

[25] A. Nunnenkamp, K. Borkje, and S. M. Girvin, SinglePhoton Optomechanics, Phys. Rev. Lett. 107, 063602 (2011).

[26] A. Nunnenkamp, K. Børkje, and S. M. Girvin, Cooling in the Single-Photon Strong-Coupling Regime of Cavity Optomechanics, Phys. Rev. A 85, 051803(R) (2012).

[27] B. He, Quantum Optomechanics beyond Linearization, Phys. Rev. A 85, 063820 (2012).

[28] T. Hong, H. Yang, H. Miao, and Y. Chen, Open Quantum Dynamics of Single-Photon Optomechanical Devices, Phys. Rev. A 88, 023812 (2013).

[29] P. D. Nation, Nonclassical Mechanical States in an Optomechanical Micromaser Analog, Phys. Rev. A 88, 053828 (2013).
[30] A. J. Rimberg, M. P. Blencowe, A. D. Armour, and P. D. Nation, A Cavity-Cooper Pair Transistor Scheme for Investigating Quantum Optomechanics in the Ultra-Strong Coupling Regime, New J. Phys. 16, 055008 (2014).

[31] A. F. Kockum, A. Miranowicz, S. De Liberato, S. Savasta, and F. Nori, Ultrastrong Coupling between Light and Matter, Nat. Rev. Phys. 1, 19 (2019).

[32] P. Forn-Díaz, L. Lamata, E. Rico, J. Kono, and E. Solano, Ultrastrong Coupling Regimes of Light-Matter Interaction, Rev. Mod. Phys. 91, 025005 (2019).

[33] K. W. Murch, K. L. Moore, S. Gupta, and D. Stamper-kurn, Observation of Quantum-Measurement Backaction with an Ultracold Atomic Gas, Nat. Phys. 4, 561 (2008).

[34] F. Brennecke, S. Ritter, T. Donner, and T. Esslinger, Cavity Optomechanics with a Bose-Einstein Condensate, Science 322, 235 (2008).

[35] R. Leijssen and E. Verhagen, Strong Optomechanical Interactions in a Sliced Photonic Crystal Nanobeam, Sci. Rep. 5, 15974 (2015).

[36] R. Leijssen, G. R. La Gala, L. Freisem, J. T. Muhonen, and E. Verhagen, Nonlinear Cavity Optomechanics with Nanomechanical Thermal Fluctuation, Nat. Commun. 8, 16024 (2017).

[37] C. Reinhardt, T. Müller, A. Bourassa, and J. C. Sankey, Ultralow-Noise SiN Trampoline Resonators for Sensing and Optomechanics, Phys. Rev. X 6, 021001 (2016).

[38] J. D. Thompson, B. M. Zwickl, A. M. Jayich, F. Marquardt, S. M. Girvin, and J. G. E. Harris, Strong Dispersive Coupling of a High-Finesse Cavity to a Micromechanical Membrane, Nature (London) 452, 72 (2008).

[39] I. Favero, S. Stapfner, D. Hunger, P. Paulitschke, J. Reichel, H. Lorenz, E. M. Weig, and K. Karrai, Fluctuating Nanomechanical System in a High Finesse Optical Microcavity, Opt. Express 17, 12813 (2009).

[40] G. Anetsberger, O. Arcizet, Q. P. Unterreithmeier, R. Rivière, A. Schliesser, E. M. Weig, J. P. Kotthaus, and T. J. Kippenberg, Near-Field Cavity Optomechanics with Nanomechanical Oscillators, Nat. Phys. 5, 909 (2009).

[41] J. C. Sankey, C. Yang, B. M. Zwickl, A. M. Jayich, and J. G. E. Harris, Strong and Tunable Nonlinear Optomechanical Coupling in a Low-Loss System, Nat. Phys. 6, 707 (2010).

[42] S. Stapfner, L. Ost, D. Hunger, J. Reichel, I. Favero, and E. M. Weig, Cavity-Enhanced Optical Detection of Carbon Nanotube Brownian Motion, Appl. Phys. Lett. 102, 151910 (2013).

[43] I. Favero, J. Sankey, and E. M. Weig, Mechanical Resonators in the Middle of an Optical Cavity (Springer, Berlin, Heidelberg, 2014), pp. 83-119, https://doi.org/10.1007/ 978-3-642-55312-7_5.

[44] N. Kiesel, F. Blaser, U. Delic, D. Grass, R. Kaltenbaek, and M. Aspelmeyer, Cavity Cooling of an Optically Levitated Nanoparticle, Proc. Natl. Acad. Sci. U.S.A. 110, 14180 (2013).

[45] A. Jöckel, A. Faber, T. Kampschulte, M. Korppi, M. T. Rakher, and P. Treutlein, Sympathetic Cooling of a Membrane Oscillator in a Hybrid Mechanical-Atomic System, Nat. Nanotechnol. 10, 55 (2015).

[46] D. J. Wilson, V. Sudhir, N. Piro, R. Schilling, A. Ghadimi, and T. J. Kippenberg, Measurement-Based Control of a 
Mechanical Oscillator at Its Thermal Decoherence Rate, Nature (London) 524, 325 (2015).

[47] O. Arcizet, V. Jacques, A. Siria, P. Poncharal, P. Vincent, and S. Seidelin, A Single Nitrogen-Vacancy Defect Coupled to a Nanomechanical Oscillator, Nat. Phys. 7, 879 (2011).

[48] A. Gloppe, P. Verlot, E. Dupont-Ferrier, A. Siria, P. Poncharal, G. Bachelier, P. Vincent, and O. Arcizet, Bidimensional Nano-Optomechanics and Topological Backaction in a Non-conservative Radiation Force Field, Nat. Nanotechnol. 9, 920 (2014).

[49] B. Pigeau, S. Rohr, L. M. de Lépinay, A. Gloppe, V. Jacques, and O. Arcizet, Observation of a Phononic Mollow Triplet in a Multimode Hybrid Spin-Nanomechanical System, Nat. Commun. 6, 8603 (2015).

[50] L. M. de Lépinay, B. Pigeau, B. Besga, P. Vincent, P. Poncharal, and $\mathrm{O}$. Arcizet, A Universal and Ultrasensitive Vectorial Nanomechanical Sensor for Imaging 2D Force Field, Nat. Nanotechnol. 12, 156 (2017).

[51] N. Rossi, F. R. Braakman, D. Cadeddu, D. Vasyukov, G. Tütüncüoglu, A.F. i Morral, and M. Poggio, Vectorial Scanning Force Microscopy Using a Nanowire Sensor, Nat. Nanotechnol. 12, 150 (2017).

[52] L. M. de Lépinay, B. Pigeau, B. Besga, and O. Arcizet, Eigenmode Orthogonality Breaking and Anomalous Dynamics in Multimode Nano-Optomechanical Systems under Non-reciprocal Coupling, Nat. Commun. 9, 1401 (2018).

[53] Y. Colombe, T. Steinmetz, G. Dubois, F. Linke, D. Hunger, and J. Reichel, Strong Atom-Field Coupling for Bose-
Einstein Condensates in an Optical Cavity on a Chip, Nature (London) 450, 272 (2007).

[54] D. Hunger, T. Steinmetz, Y. Colombe, C. Deutsch, T. W. Hänsch, and J. Reichel, A Fiber Fabry-Perot Cavity with High Finesse, New J. Phys. 12, 065038 (2010).

[55] M. Mader, J. Reichel, T. W. Hänsch, and D. Hunger, A Scanning Cavity Microscope, Nat. Commun. 6, 7249 (2015).

[56] F. Ferri, S. Garcia, M. Baghdad, J. Reichel, and R. Long, Mapping Standing-Wave Cavity Modes with a Commercial Scanning Near-Field Microscope Tip, Rev. Sci. Instrum. 91, 033104 (2020).

[57] C. F. Bohren and D. Huffman, Absorption and Scattering of Light by Small Particles (Wiley VCH, Berlin, 1983).

[58] A. Reigue et al., Cavity Nano-Ptomechanics with Suspended Nanowires: Modelisation and Novel Phenomenology (to be published).

[59] D. Hunger, C. Deutsch, R. J. Barbour, R. J. Warburton, and J. Reichel, Laser Micro-Fabrication of Concave, Low-Roughness Features in Silica, AIP Adv. 2, 012119 (2012).

[60] F. Fogliano et al., Ultrasensitive Nano-Optomechanical Force Sensor at Dilution Temperatures, arXiv:2009.02912.

[61] https://pyrpl.readthedocs.io/en/latest/

[62] A. M. Jayich, J. C. Sankey, B. M. Zwickl, C. Yang, J. D. Thompson, S. M. Girvin, A. A. Clerk, F. Marquardt, and J. G. E. Harris, Dispersive Optomechanics: A Membrane Inside a Cavity, New J. Phys. 10, 095008 (2008). 